\title{
QUANTUM INTEGRALS OF MOTION FOR VARIABLE QUADRATIC HAMILTONIANS
}

\author{
RICARDO CORDERO-SOTO, ERWIN SUAZO, AND SERGEI K. SUSLOV
}

\begin{abstract}
We construct integrals of motion for several models of the quantum damped oscillators in a framework of a general approach to the time-dependent Schrödinger equation with variable quadratic Hamiltonians. An extension of the Lewis-Riesenfeld dynamical invariant is given. The time-evolution of the expectation values of the energy related positive operators is determined for the oscillators under consideration. A proof of uniqueness of the corresponding Cauchy initial value problem is discussed as an application.
\end{abstract}

\section{An Introduction}

Evolution of a nonrelativistic quantum system from a given initial state to the final state is governed by the (time-dependent) Schrödinger equation. Unfortunately, its explicit solutions are available only for the simplest Hamiltonians and, in general, one has to rely on a variety of approximation, asymptotic and numerical methods. Luckily among the integrable cases are the so-called quadratic Hamiltonians that attracted substantial attention over the years in view of their great importance to many advanced quantum problems. Examples can be found in quantum and physical optics [34], [74], [114], [116], physics of lasers and masers [128], [142], 131], [148, molecular spectroscopy [41], quantum chemistry, quantization of mechanical systems [31, [45], [46], [47], [50, [75], [77] and Hamiltonian cosmology [9], [51], [52], [58], [64], [114], [124], [125], [126]. They include coherent states [95], [96], [97], [74] and Berry's phase [7], [8], [18], [57], 84], [105], asymptotic and numerical methods [54], 68, [78], [103], [107], charged particle traps [94 and motion in uniform magnetic fields [26], [29], 39], [80], [88, [89], [91], 97], polyatomic molecules in varying external fields, crystals through which an electron is passing and exciting the oscillator modes and other interactions of the modes with external fields [50]. Quadratic Hamiltonians have particular applications in quantum electrodynamics because the electromagnetic field can be represented as a set of forced harmonic oscillators [10], [50], [39], [53], [65], and [102]. Nonlinear oscillators play a central role in the novel theory of Bose-Einstein condensation [30] based on the nonlinear Schrödinger (or Gross-Pitaevskii) equation [66], 67], [73], [119].

The one-dimensional Schrödinger equation with variable quadratic Hamiltonians of the form

$$
i \frac{\partial \psi}{\partial t}=-a(t) \frac{\partial^{2} \psi}{\partial x^{2}}+b(t) x^{2} \psi-i\left(c(t) x \frac{\partial \psi}{\partial x}+d(t) \psi\right),
$$

Date: October 16, 2018.

1991 Mathematics Subject Classification. Primary 81Q05, 35C05. Secondary 42A38.

Key words and phrases. The time-dependent Schrödinger equation, Cauchy initial value problem, Green function, propagator, quantum damped oscillators, Caldirola-Kanai Hamiltonians, quantum integrals of motion, LewisRiesenfeld dynamical invariant, Ermakov's equation, Ehrenfest's theorem. 
where $a(t), b(t), c(t)$, and $d(t)$ are real-valued functions of time $t$ only, can be integrated in the following manner (see, for example, [26], 27], [28], [36], [81, [93, [101, [134, [135, [136, [137], [149], and [150] for a general approach and some elementary solutions). The Green functions, or Feynman's propagators, are given by [26], [136]:

$$
\psi=G(x, y, t)=\frac{1}{\sqrt{2 \pi i \mu(t)}} e^{i\left(\alpha(t) x^{2}+\beta(t) x y+\gamma(t) y^{2}\right)},
$$

where

$$
\begin{aligned}
& \alpha(t)=\frac{1}{4 a(t)} \frac{\mu^{\prime}(t)}{\mu(t)}-\frac{d(t)}{2 a(t)}, \\
& \beta(t)=-\frac{h(t)}{\mu(t)}, \quad h(t)=\exp \left(-\int_{0}^{t}(c(\tau)-2 d(\tau)) d \tau\right), \\
& \gamma(t)=\frac{a(t) h^{2}(t)}{\mu(t) \mu^{\prime}(t)}+\frac{d(0)}{2 a(0)}-4 \int_{0}^{t} \frac{a(\tau) \sigma(\tau) h^{2}(\tau)}{\left(\mu^{\prime}(\tau)\right)^{2}} d \tau
\end{aligned}
$$

and the function $\mu(t)$ satisfies the so-called characteristic equation

$$
\mu^{\prime \prime}-\tau(t) \mu^{\prime}+4 \sigma(t) \mu=0
$$

with

$$
\tau(t)=\frac{a^{\prime}}{a}-2 c+4 d, \quad \sigma(t)=a b-c d+d^{2}+\frac{d}{2}\left(\frac{a^{\prime}}{a}-\frac{d^{\prime}}{d}\right)
$$

subject to the initial data

$$
\mu(0)=0, \quad \mu^{\prime}(0)=2 a(0) \neq 0 .
$$

(More details can be found in Refs. [26], [136] and a Hamiltonian structure is considered in Refs. [7], [28.) Then, by the superposition principle, solution of the Cauchy initial value problem can be presented in an integral form

$$
\psi(x, t)=\int_{-\infty}^{\infty} G(x, y, t) \varphi(y) d y, \quad \lim _{t \rightarrow 0^{+}} \psi(x, t)=\varphi(x)
$$

for a suitable initial function $\varphi$ on $\mathbb{R}$ (a rigorous proof is given in Ref. [136] and uniqueness is analyzed in this paper).

We discuss integrals of motion for several particular models of the damped and generalized quantum oscillators. The simple harmonic oscillator is of interest in many quantum problems [50], [80], [102], and [129]. The forced harmonic oscillator was originally considered by Richard Feynman in his path integrals approach to the nonrelativistic quantum mechanics [46], [47, [48, [49], and [50]; see also [93]. Its special and limiting cases were discussed in Refs. [6], [53], [61], [100], [102], [144] for the simple harmonic oscillator and in Refs. [3], [12], 60], [108], [123] for the particle in a constant external field; see also references therein. The damped oscillations have been studied to a great extent in classical mechanics [4, [5] and [79]. Their quantum analogs are introduced and analyzed from different viewpoints by many authors; see, for example, [13], [19], [23], [24], [25], [27], [32], [33], [35], [37], [40], [82], [83], [69], [104], [110], [139], [140], [143], [146], and references therein. The quantum parametric oscillator with variable frequency is also largely studied in view of its physical importance; see, for example, [22], [39], [62], 663], [81], [95], [97], [117], [118], [121], [122], [130], and [133]; a detailed bibliography is given in [14]. 
In the present paper we revisit a familiar topic of the quantum integrals of motion for the timedependent Schrödinger equation

$$
i \frac{\partial \psi}{\partial t}=H(t) \psi
$$

with variable quadratic Hamiltonians of the form

$$
H=a(t) p^{2}+b(t) x^{2}+d(t)(p x+x p),
$$

where $p=-i \partial / \partial x, \hbar=1$ and $a(t), b(t), c(t)=2 d(t)$ are some real-valued functions of time only (see, for example, [36], [84], [91], [97], 98], [149], [150] and references therein). A related energy operator $E$ is defined in a traditional way as a quadratic in $p$ and $x$ operator that has constant expectation values [39]:

$$
\frac{d}{d t}\langle E\rangle=\frac{d}{d t} \int_{-\infty}^{\infty} \psi^{*} E \psi d x=0
$$

It is well-known that such quadratic invariants are not unique. Although an elegant general solution is known, say, for the parametric oscillator, it involves an integration of nonlinear Ermakov's equation [91. Here the simplest energy operators are constructed for several integrable models of the damped and modified quantum oscillators. Then an extension of the familiar Lewis-Riesenfeld quadratic invariant is given to the most general case of the variable non-self-adjoint quadratic Hamiltonian (see also [84], [149], [150], we do not use canonical transformations and deal only with real-valued solutions of the corresponding generalized Ermakov system), which seems to be missing in the available literature and may be considered as the main result of this paper. (An attempt to collect relevant references is made1.) Group-theoretical aspects will be discussed elsewhere, we only provide the factorization of the general quadratic invariant (see also [138]).

In general the average $\langle E\rangle$ is not positive. A complete dynamics of the expectation values of some energy-related positive operators is found instead for each model, which is a somewhat interesting result on its own. In addition to other works [7], [39], [36], [57], [91, [95], 98], [149], [150] these advances allow us to discuss uniqueness of the corresponding Cauchy initial value problem for the special models and for the general quadratic Hamiltonian under consideration as a modest contribution to this well-developed area of quantum mechanics and partial differential equations. Further relations of the quadratic invariants with the solution of the initial value problem are discussed in the forthcoming paper 138 .

The paper is organized as follows. In Section 2 we review several exactly solvable models of the damped and generalized oscillators in quantum mechanics. Some of these "exotic" oscillators with variable quadratic Hamiltonians appear to be missing, and/or are just recently introduced, in the available literature. The corresponding Green functions are found in terms of elementary functions. The dynamical invariants and quadratic energy-related operators are discussed in Sections 3 and 4. The last section is concerned with an application to the Cauchy initial value problems. The classical equations of motion for the expectation values of the position operator for the quantum oscillators under consideration are derived in Appendix A. The Heisenberg uncertainty relation and linear dynamic invariants are revisited, respectively, in Appendices B and C. Solutions of a required differential equation are given in Appendix D to make our presentation is as self-contained as possible.

\footnotetext{
${ }^{1} \mathrm{~A}$ complete bibliography on classical and quantum generalized harmonic oscillators, their invariants, grouptheoretical methods and applications is very extensive. Only case of the damped oscillators in 33 includes about 600 references!
} 


\section{Some Integrable Quadratic Hamiltonians}

Quantum systems with the Hamiltonians (1.11) are called the generalized harmonic oscillators [7], [36], [57], [84], [149], [150]. In this paper we concentrate, among others, on the following variable Hamiltonians: the Caldirola-Kanai Hamiltonian of the quantum damped oscillator [13], [33], 69], [146] and some of its natural modifications, a modified oscillator introduced by Meiler, CorderoSoto and Suslov [101], [28], the quantum damped oscillator of Chruściński and Jurkowski [25] in the coordinate and momentum representations and a quantum-modified parametric oscillator which is believed to be new. The Green functions are derived in a united way.

2.1. The Caldirola-Kanai Hamiltonian. A model of the quantum damped oscillator with a variable Hamiltonian of the form

$$
H=\frac{\omega_{0}}{2}\left(e^{-2 \lambda t} p^{2}+e^{2 \lambda t} x^{2}\right)
$$

is called the Caldirola-Kanai model [4], [13], [33], 69], [146]. Nowadays it is a standard way of adding friction to the quantum harmonic oscillator. The Green function is given by

$$
G(x, y, t)=\sqrt{\frac{\omega e^{\lambda t}}{2 \pi i \omega_{0} \sin \omega t}} e^{i\left(\alpha(t) x^{2}+\beta(t) x y+\gamma(t) y^{2}\right)}, \quad \omega=\sqrt{\omega_{0}^{2}-\lambda^{2}}>0,
$$

where

$$
\begin{aligned}
& \alpha(t)=\frac{\omega \cos \omega t-\lambda \sin \omega t}{2 \omega_{0} \sin \omega t} e^{2 \lambda t}, \\
& \beta(t)=-\frac{\omega}{\omega_{0} \sin \omega t} e^{\lambda t}, \\
& \gamma(t)=\frac{\omega \cos \omega t+\lambda \sin \omega t}{2 \omega_{0} \sin \omega t} .
\end{aligned}
$$

This popular model had been studied in detail by many authors from different viewpoints; see, for example, [2], [11, [15], [16], [17], 20], 21], [37], [70], [71], [72, [75], [77], 82], [110], [112], [115], [127], 139], [140], [145], [151] and references therein, a detailed bibliography can be found in [33], [146].

2.2. A Modified Caldirola-Kanai Hamiltonian. In this paper, we would like to consider another version of the quantum damped oscillator with variable Hamiltonian of the form

$$
H=\frac{\omega_{0}}{2}\left(e^{-2 \lambda t} p^{2}+e^{2 \lambda t} x^{2}\right)-\lambda(p x+x p) .
$$

The Green functions in (2.2) has

$$
\begin{aligned}
& \alpha(t)=\frac{\omega \cos \omega t+\lambda \sin \omega t}{2 \omega_{0} \sin \omega t} e^{2 \lambda t}, \\
& \beta(t)=-\frac{\omega}{\omega_{0} \sin \omega t} e^{\lambda t}, \\
& \gamma(t)=\frac{\omega \cos \omega t-\lambda \sin \omega t}{2 \omega_{0} \sin \omega t} .
\end{aligned}
$$

This can be derived directly from equations (1.2) $-(1.8)$ following Refs. [26] and [27]. 
The Ehrenfest theorem for both Caldirola-Kanai models has the same form

$$
\frac{d^{2}}{d t^{2}}\langle x\rangle+2 \lambda \frac{d}{d t}\langle x\rangle+\omega_{0}^{2}\langle x\rangle=0
$$

which coincides with the classical equation of motion for a damped oscillator [5], [79]. Details are provided in Appendix A.

2.3. The United Model. The following non-self-adjoint Hamiltonian:

$$
H=\frac{\omega_{0}}{2}\left(e^{-2 \lambda t} p^{2}+e^{2 \lambda t} x^{2}\right)-\mu x p
$$

coincides with the original Caldirola-Kanai model when $\mu=0$ and the Hamiltonian is self-adjoint. Another special case $\lambda=0$ corresponds to the quantum damped oscillator discussed in [27] as an example of a simple quantum system with the non-self-adjoint Hamiltonian. (This is an alternative way to introduce dissipation of energy to the quantum harmonic oscillator.) Combining both cases we refer to (2.11) as the united Hamiltonian.

The Green function is given by

$$
G(x, y, t)=\sqrt{\frac{\omega e^{(\lambda-\mu) t}}{2 \pi i \omega_{0} \sin \omega t}} e^{i\left(\alpha(t) x^{2}+\beta(t) x y+\gamma(t) y^{2}\right)},
$$

where

$$
\begin{aligned}
\alpha(t) & =\frac{\omega \cos \omega t+(\mu-\lambda) \sin \omega t}{2 \omega_{0} \sin \omega t} e^{2 \lambda t}, \\
\beta(t) & =-\frac{\omega}{\omega_{0} \sin \omega t} e^{\lambda t} \\
\gamma(t) & =\frac{\omega \cos \omega t+(\lambda-\mu) \sin \omega t}{2 \omega_{0} \sin \omega t}
\end{aligned}
$$

with $\omega=\sqrt{\omega_{0}^{2}-(\lambda-\mu)^{2}}>0$.

In this case the Ehrenfest theorem takes the form:

$$
\frac{d^{2}}{d t^{2}}\langle x\rangle+2(\lambda+\mu) \frac{d}{d t}\langle x\rangle+\left(\omega_{0}^{2}+4 \lambda \mu\right)\langle x\rangle=0 .
$$

It is derived in Appendix A and the Heisenberg uncertainty relation is discussed in Appendix B.

2.4. A Modified Oscillator. The one-dimensional Hamiltonian of a modified oscillator introduced by Meiler, Cordero-Soto and Suslov [101, 28] has the form

$$
\begin{aligned}
H & =(\cos t p+\sin t x)^{2} \\
& =\cos ^{2} t p^{2}+\sin ^{2} t x^{2}+\sin t \cos t(p x+x p) \\
& =\frac{1}{2}\left(p^{2}+x^{2}\right)+\frac{1}{2} \cos 2 t\left(p^{2}-x^{2}\right)+\frac{1}{2} \sin 2 t(p x+x p) .
\end{aligned}
$$


(A physical interpretation of this Hamiltonian from the viewpoint of quantum dynamical invariants will be discussed in Section 4.) The Green function is given in terms of trigonometric and hyperbolic functions as follows

$$
\begin{aligned}
G(x, y, t)= & \frac{1}{\sqrt{2 \pi i(\cos t \sinh t+\sin t \cosh t)}} \\
& \times \exp \left(\frac{\left(x^{2}-y^{2}\right) \sin t \sinh t+2 x y-\left(x^{2}+y^{2}\right) \cos t \cosh t}{2 i(\cos t \sinh t+\sin t \cosh t)}\right) .
\end{aligned}
$$

More details can be found in [101], 28]. The corresponding Ehrenfest theorem, namely,

$$
\frac{d^{2}}{d t^{2}}\langle x\rangle+2 \tan t \frac{d}{d t}\langle x\rangle-2\langle x\rangle=0
$$

is derived in Appendix A.

2.5. The Modified Damped Oscillator. The time-dependent Schrödinger equation

$$
i \hbar \frac{\partial \psi}{\partial t}=H(t) \psi
$$

with the variable quadratic Hamiltonian of the form

$$
H=\frac{p^{2}}{2 m \cosh ^{2}(\lambda t)}+\frac{m \omega_{0}^{2}}{2} \cosh ^{2}(\lambda t) x^{2}, \quad p=\frac{\hbar}{i} \frac{\partial}{\partial x}
$$

has been recently considered by Chruściński and Jurkowski [25] as a model of the quantum damped oscillator; see also [106].

In this case the characteristic equation (1.6) takes the form

$$
\mu^{\prime \prime}+2 \lambda \tanh (\lambda t) \mu^{\prime}+\omega_{0}^{2} \mu=0 .
$$

The particular solution is given by

$$
\mu(t)=\frac{\hbar}{m \omega} \frac{\sin (\omega t)}{\cosh (\lambda t)}, \quad \omega=\sqrt{\omega_{0}^{2}-\lambda^{2}}>0
$$

and the corresponding propagator can be presented as follows

$$
G(x, y, t)=\sqrt{\frac{m \omega \cosh (\lambda t)}{2 \pi i \hbar \sin (\omega t)}} e^{i\left(\alpha(t) x^{2}+\beta(t) x y+\gamma(t) y^{2}\right)},
$$

where

$$
\begin{gathered}
\alpha(t)=\frac{m \cosh (\lambda t)}{2 \hbar \sin (\omega t)}(\omega \cos (\omega t) \cosh (\lambda t)-\lambda \sin (\omega t) \sinh (\lambda t)), \\
\beta(t)=-\frac{m \omega \cosh (\lambda t)}{2 \hbar \sin (\omega t)}, \\
\gamma(t)=\frac{m \omega \cos (\omega t)}{2 \hbar \sin (\omega t)} .
\end{gathered}
$$


(We somewhat simplify the original propagator found in [25]; see also [76].) This Green function can be independently derived from our equations (1.3)-(1.5) with the help of the following elementary antiderivative:

$$
\begin{aligned}
& \left(\frac{\lambda \cos (\omega t+\delta) \sinh (\lambda t)+\omega \sin (\omega t+\delta) \cosh (\lambda t)}{\omega \cos (\omega t+\delta) \cosh (\lambda t)-\lambda \sin (\omega t+\delta) \sinh (\lambda t)}\right)^{\prime} \\
& \quad=\frac{\omega \omega_{0}^{2} \cosh ^{2}(\lambda t)}{(\omega \cos (\omega t+\delta) \cosh (\lambda t)-\lambda \sin (\omega t+\delta) \sinh (\lambda t))^{2}}
\end{aligned}
$$

Further details are left to the reader.

Special cases are as follows: when $\lambda=0$, one recovers the standard propagator for the linear harmonic oscillator [50], and $\omega_{0}=0$ gives a pure damping case [76]:

$$
G(x, y, t)=\sqrt{\frac{m \lambda}{2 \pi i \hbar \tanh (\omega t)}} \exp \left(\frac{i m \lambda(x-y)^{2}}{2 \hbar \tanh (\omega t)}\right) .
$$

In the limit $\lambda \rightarrow 0$ formula (2.29) reproduces the propagator for a free particle [50].

The Ehrenfest theorem for the quantum damped oscillator of Chruściński and Jurkowski coincides with our characteristic equation (2.22); see Appendix A for more details.

It is worth adding that in the momentum representation, when $p \leftrightarrow x$, a rescaled Hamiltonian (2.21) $\left(\hbar=m \omega_{0}=1\right)$ takes the form

$$
H=\frac{\omega_{0}}{2}\left(\cosh ^{2}(\lambda t) p^{2}+\frac{x^{2}}{\cosh ^{2}(\lambda t)}\right) .
$$

The corresponding characteristic equation

$$
\mu^{\prime \prime}-2 \lambda \tanh (\lambda t) \mu^{\prime}+\omega_{0}^{2} \mu=0
$$

has a required elementary solution

$$
\mu=\frac{1}{\omega_{0}}(\lambda \cos (\omega t) \sinh (\lambda t)+\omega \sin (\omega t) \cosh (\lambda t))
$$

with $\mu^{\prime}(0)=2 a(0)=\omega_{0}$ and

$$
\mu \rightarrow \frac{1}{2 \omega_{0}} e^{\lambda t}(\lambda \cos (\omega t)+\omega \sin (\omega t))
$$

as $t \rightarrow \infty$. The Green function is given by formula (1.2) with the following coefficients:

$$
\begin{aligned}
& \alpha(t)=\frac{\omega_{0} \cos (\omega t)}{2 \cosh (\lambda t)(\lambda \cos (\omega t) \sinh (\lambda t)+\omega \sin (\omega t) \cosh (\lambda t))}, \\
& \beta(t)=-\frac{\omega_{0}}{\lambda \cos (\omega t) \sinh (\lambda t)+\omega \sin (\omega t) \cosh (\lambda t)} \\
& \gamma(t)=\frac{\omega_{0}(\omega \cos (\omega t) \cosh (\lambda t)-\lambda \sin (\omega t) \sinh (\lambda t))}{2 \omega(\lambda \cos (\omega t) \sinh (\lambda t)+\omega \sin (\omega t) \cosh (\lambda t))}
\end{aligned}
$$

The details are left to the reader. 
2.6. A Modified Parametric Oscillator. In a similar fashion we consider the following Hamiltonian:

$$
\begin{aligned}
H= & \frac{\omega}{2}\left(\tanh ^{2}(\lambda t+\delta) p^{2}+\operatorname{coth}^{2}(\lambda t+\delta) x^{2}\right) \\
& +\frac{\lambda}{\sinh (2 \lambda t+2 \delta)}(p x+x p) \quad(\delta \neq 0),
\end{aligned}
$$

which seems to be missing in the available literature. The corresponding characteristic equation:

$$
\mu^{\prime \prime}-\frac{4 \lambda}{\sinh (2 \lambda t+2 \delta)} \mu^{\prime}+\left(\omega^{2}+\frac{2 \lambda^{2}}{\sinh ^{2}(\lambda t+\delta)}\right) \mu=0
$$

has an elementary solution of the form:

$$
\mu=\sin (\omega t) \frac{\tanh (\lambda t+\delta)}{\operatorname{coth} \delta} .
$$

In the limit $t \rightarrow \infty, \mu \rightarrow \sin (\omega t) \tanh \delta$.

The Green function can be found as follows

$$
G(x, y, t)=\sqrt{\frac{\operatorname{coth} \delta}{2 \pi i \sin (\omega t) \tanh (\lambda t+\delta)}} e^{i\left(\alpha(t) x^{2}+\beta(t) x y+\gamma(t) y^{2}\right)},
$$

where

$$
\begin{gathered}
\alpha(t)=\frac{1}{2} \cot (\omega t) \operatorname{coth}^{2}(\lambda t+\delta), \\
\beta(t)=-\frac{\operatorname{coth} \delta}{\sin (\omega t)} \operatorname{coth}(\lambda t+\delta), \\
\gamma(t)=\frac{1}{2} \cot (\omega t) \operatorname{coth}^{2} \delta .
\end{gathered}
$$

The Ehrenfest theorem coincides with the characteristic equation (2.38). One should interchange $a \leftrightarrow b$ and $d \rightarrow-d$ in the momentum representation [28]. The corresponding solutions can be found with the help of the substitution $\delta \rightarrow \delta+i \pi / 2$. The trigonometric cases, when $\lambda \rightarrow i \lambda, \delta \rightarrow i \delta$ and $\omega \rightarrow-\omega$, are left to the reader.

2.7. Parametric Oscillators. In conclusion a somewhat related quantum parametric oscillator:

$$
H=\frac{1}{2}\left(p^{2}+\left(\omega^{2}+\frac{2 \lambda^{2}}{\cosh ^{2}(\lambda t)}\right) x^{2}\right)
$$

when

$$
\mu^{\prime \prime}+\left(\omega^{2}+\frac{2 \lambda^{2}}{\cosh ^{2}(\lambda t)}\right) \mu=0
$$

and

$$
\mu=\frac{\lambda \cos (\omega t) \sinh (\lambda t)+\omega \sin (\omega t) \cosh (\lambda t)}{\left(\omega^{2}+\lambda^{2}\right) \cosh (\lambda t)},
$$

has the Green function (1.2) with the following coefficients:

$$
\alpha(t)=\frac{\left(\omega^{2}+\lambda^{2} \cosh ^{-2}(\lambda t)\right) \cos (\omega t)-\lambda \omega \tanh (\lambda t) \sin (\omega t)}{2(\omega \sin (\omega t)+\lambda \tanh (\lambda t) \cos (\omega t))},
$$




$$
\begin{aligned}
& \beta(t)=-\frac{\omega^{2}+\lambda^{2}}{\omega \sin (\omega t)+\lambda \tanh (\lambda t) \cos (\omega t)}, \\
& \gamma(t)=\frac{\left(\omega^{2}+\lambda^{2}\right)(\omega \cos (\omega t)-\lambda \tanh (\lambda t) \sin (\omega t))}{2 \omega(\omega \sin (\omega t)+\lambda \tanh (\lambda t) \cos (\omega t))} .
\end{aligned}
$$

The Green function for the parametric oscillator in general:

$$
H=\frac{1}{2}\left(p^{2}+\omega^{2}(t) x^{2}\right)
$$

can be found, for example, in Ref. [81]. (The time-dependent quantum oscillator was thoroughly examined by Husimi [62, 63] and later many authors had treated different aspects of the problem; see [39], [95], [97], [98], [117], [118], [121], [122], [130] and [133]; a detailed bibliography is given in Ref. [14.)

\section{Expectation Values of Quadratic Operators}

We start from a convenient differentiation formula.

Lemma 1. Let

$$
\begin{aligned}
& H=a(t) p^{2}+b(t) x^{2}+d(t)(p x+x p), \\
& O=A(t) p^{2}+B(t) x^{2}+C(t)(p x+x p)
\end{aligned}
$$

and

$$
\langle O\rangle=\langle\psi, O \psi\rangle=\int_{-\infty}^{\infty} \psi^{*} O \psi d x, \quad i \frac{\partial \psi}{\partial t}=H \psi
$$

(we use the star for complex conjugate). Then

$$
\begin{aligned}
\frac{d}{d t}\langle O\rangle= & \left(\frac{d A}{d t}+4(a C-d A)\right)\left\langle p^{2}\right\rangle \\
& +\left(\frac{d B}{d t}+4(d B-b C)\right)\left\langle x^{2}\right\rangle \\
& +\left(\frac{d C}{d t}+2(a B-b A)\right)\langle p x+x p\rangle .
\end{aligned}
$$

Proof. The time derivative of the expectation value can be written as [80, [102], [129]:

$$
\frac{d}{d t}\langle O\rangle=\left\langle\frac{\partial O}{\partial t}\right\rangle+\frac{1}{i}\langle[O, H]\rangle
$$

where $[O, H]=O H-H O$ (we freely interchange differentiation and integration throughout the paper, it can be justified for certain classes of solutions [92], [113], [119], [147]). One should make use of the standard commutator properties, including familiar identities

$$
\begin{array}{ll}
{\left[x^{2}, p^{2}\right]=2 i(p x+x p),} & {\left[x, p^{2}\right]=2 i p, \quad\left[x^{2}, p\right]=2 i x,} \\
{\left[p x+x p, p^{2}\right]=4 i p^{2},} & {\left[x^{2}, p x+x p\right]=4 i x^{2},}
\end{array}
$$

in order to complete the proof. 
Quantum systems with the self-adjoint Hamiltonians (3.1) are called the generalized harmonic oscillators [7], [36], [57], [84], [149], [150]. At the same time one has to deal with non-self-adjoint Hamiltonians in the theory of dissipative quantum systems (see, for example, [27], [33], [77], [143], [146] and references therein) or when using separation of variables in an accelerating frame of reference for a charged particle moving in an uniform time-dependent magnetic field [26]. An extension to the case of non-self-adjoint Hamiltonians is as follows.

Lemma 2. If

$$
\begin{gathered}
H=a(t) p^{2}+b(t) x^{2}+c(t) p x+d(t) x p \\
O=A(t) p^{2}+B(t) x^{2}+C(t) p x+D(t) x p
\end{gathered}
$$

then

$$
\begin{aligned}
\frac{d}{d t}\langle O\rangle= & \left(\frac{d A}{d t}+2 a(C+D)-(3 c+d) A\right)\left\langle p^{2}\right\rangle \\
& +\left(\frac{d B}{d t}-2 b(C+D)+(c+3 d) B\right)\left\langle x^{2}\right\rangle \\
& +\left(\frac{d C}{d t}+2(a B-b A)-(c-d) C\right)\langle p x\rangle \\
& +\left(\frac{d D}{d t}+2(a B-b A)-(c-d) D\right)\langle x p\rangle .
\end{aligned}
$$

Proof. One should use

$$
\frac{d}{d t}\langle O\rangle=\left\langle\frac{\partial O}{\partial t}\right\rangle+\frac{1}{i}\left\langle O H-H^{\dagger} O\right\rangle,
$$

where $H^{\dagger}$ is the Hermitian adjoint of the Hamiltonian operator $H$. Our formula is a simple extension of the well-known expression [80], [102], [129] to the case of a non-self-adjoint Hamiltonian [27]. Standard commutator evaluations complete the proof.

Polynomial operators of the higher orders in $x$ and $p$ can be differentiated in a similar fashion. An analog of the product rule is given in [138]. The details are left to the reader.

\section{ENERGy OpERATORS AND QUADRATIC INVARIANTS}

In the case of the time-independent Hamiltonian, one gets

$$
\frac{d}{d t}\langle H\rangle=0
$$

by (3.5). The law of conservation of energy states that

$$
E=\langle H\rangle=\text { constant }
$$

In general one has to construct quantum integrals of motion, or dynamical invariants, that are different from the variable Hamiltonian (see, for example, [91], [149], [150]; linear case is dealt with in [37], 39], [95], [98] and Appendix C). 
4.1. Energy Operators. A familiar definition is in order (see, for example, [39], [95]).

Definition 1. We call the quadratic operator (3.2) an energy operator E, or a quadratic (dynamical) invariant, if

$$
\frac{d}{d t}\langle E\rangle=0
$$

for the corresponding variable Hamiltonian (3.1).

By Lemma 1 the coefficients of an energy operator,

$$
E=A(t) p^{2}+B(t) x^{2}+C(t)(p x+x p),
$$

must satisfy the system of ordinary differential equations:

$$
\begin{aligned}
& \frac{d A}{d t}+4(a(t) C-d(t) A)=0 \\
& \frac{d B}{d t}+4(d(t) B-b(t) C)=0 \\
& \frac{d C}{d t}+2(a(t) B-b(t) A)=0 .
\end{aligned}
$$

In general a unique solution of this system with respect to arbitrary initial conditions $A_{0}=A(0)$, $B_{0}=B(0), C_{0}=C(0)$ 59] determines a three-parameter family of the quadratic invariants (4.4). Special cases, when solutions can be found explicitly, are of the most practical importance.

In this section we find the simplest energy operators for all quadratic models under consideration as follows:

$$
\begin{aligned}
& E=\frac{\omega_{0}}{2}\left(e^{-2 \lambda t} p^{2}+e^{2 \lambda t} x^{2}\right)+\frac{\lambda}{2}(p x+x p), \\
& E=\frac{\omega_{0}}{2}\left(e^{-2 \lambda t} p^{2}+e^{2 \lambda t} x^{2}\right)-\frac{\lambda}{2}(p x+x p), \\
& E=\frac{1}{2} \cos 2 t\left(p^{2}-x^{2}\right)+\frac{1}{2} \sin 2 t(p x+p x), \\
& E=\tanh ^{2}(\lambda t+\delta) p^{2}+\operatorname{coth}^{2}(\lambda t+\delta) x^{2}
\end{aligned}
$$

for the Caldirola-Kanai Hamiltonian (2.1) [139], the modified Caldirola-Kanai Hamiltonian (2.6), the modified oscillator of Meiler, Cordero-Soto and Suslov (2.17) and for the modified parametric oscillator (2.37), respectively. Their coefficients solve the corresponding systems (4.5)-(4.7) for special initial data.

An energy operator for the united model (2.11) is given by

$$
E=\frac{\omega_{0}}{2} e^{\mu t}\left(e^{-2 \lambda t} p^{2}+e^{2 \lambda t} x^{2}\right)+\frac{1}{2}(\lambda-\mu) e^{\mu t}(p x+x p) .
$$

One should use Lemma 2; verification is left to the reader. Finally an energy operator for the quantum damped oscillator of Chruściński and Jurkowski with a rescaled Hamiltonian (5.39) is given by expression (5.40). A general case of the variable quadratic Hamiltonian is discussed in Theorem 1. 
4.2. The Lewis-Riesenfeld Invariant. Classical Hamiltonian of the generalized harmonic oscillator can be transformed into the Hamiltonian of a parametric oscillator [7], [57], [114], [150]. All quadratic invariants of the quantum parametric oscillator (2.50) can be found as follows [88], [89], [90], [91]. The corresponding system,

$$
\begin{aligned}
& A^{\prime}+2 C=0, \\
& B^{\prime}-2 \omega^{2}(t) C=0, \\
& C^{\prime}+B-\omega^{2}(t) A=0,
\end{aligned}
$$

is integrated by the substitution $A=\kappa^{2}$. Then $C=-\kappa \kappa^{\prime}, B=\kappa \kappa^{\prime \prime}+\left(\kappa^{\prime}\right)^{2}+\omega^{2}(t) \kappa^{2}$ and equation (4.14) becomes

$$
\begin{aligned}
& \left(\kappa \kappa^{\prime \prime}+\left(\kappa^{\prime}\right)^{2}+\omega^{2}(t) \kappa^{2}\right)^{\prime}+2 \omega^{2}(t) \kappa \kappa^{\prime}=0 \\
& \kappa\left(\kappa^{\prime \prime}+\omega^{2}(t) \kappa\right)^{\prime}+3 \kappa^{\prime}\left(\kappa^{\prime \prime}+\omega^{2}(t) \kappa\right)=0
\end{aligned}
$$

or with an integrating factor:

(see [91] and [86]). Thus

$$
\frac{d}{d t}\left(\kappa^{3}\left(\kappa^{\prime \prime}+\omega^{2}(t) \kappa\right)\right)=0
$$

$$
\kappa^{\prime \prime}+\omega^{2}(t) \kappa=\frac{c_{0}}{\kappa^{3}} \quad\left(c_{0}=0,1\right)
$$

and a general solution of the system (4.13)-(4.15) is given by

$$
A=\kappa^{2}, \quad B=\left(\kappa^{\prime}\right)^{2}+\frac{c_{0}}{\kappa^{2}}, \quad C=-\kappa \kappa^{\prime}
$$

in terms of solutions of the nonlinear equation (4.17), which is called Ermakov's equation, when $c_{0}=1$ [44] (see also, [85], [90], [120] and [130]). Thus the quadratic integrals of motion can be presented in the form [91]:

$$
E=\left(\kappa p-\kappa^{\prime} x\right)^{2}+\frac{c_{0}}{\kappa^{2}} x^{2}
$$

for any given solution of the Ermakov equation (4.17). This quantum invariant is an analog of the Ermakov-Lewis integral of motion for the classical parametric oscillator [44], 88], [89], [90], [141].

In general if two linearly independent solutions of the classical parametric oscillator equation are available:

$$
u^{\prime \prime}+\omega^{2}(t) u=0, \quad v^{\prime \prime}+\omega^{2}(t) v=0,
$$

then solutions of the nonlinear Ermakov equation:

$$
\kappa^{\prime \prime}+\omega^{2}(t) \kappa=\frac{1}{\kappa^{3}}
$$

are given by

$$
\kappa=\left(A u^{2}+2 B u v+C v^{2}\right)^{1 / 2}
$$

(so-called Pinney's solution [120, [43], [85, [90], [114]), where the constants $A, B$ and $C$ are related according to $A C-B^{2}=1 / W^{2}$ with $W$ being the constant Wronskian of the two linearly independent solutions.

For example, in the case of the simple harmonic oscillator with $\omega(t)=1$, there are two elementary solutions:

$$
\kappa=1 \quad\left(c_{0}=1\right), \quad \kappa=\cos t \quad\left(c_{0}=0\right)
$$


and the energy operators are given by

$$
\begin{aligned}
H & =\frac{1}{2}\left(p^{2}+x^{2}\right), \\
E & =(\cos t p+\sin t x)^{2} .
\end{aligned}
$$

It provides a somewhat better understanding of the nature of the Hamiltonian discussed by Meiler, Cordero-Soto and Suslov [101] — this operator plays a role of the simplest time-dependent quadratic integral of motion for the linear harmonic oscillator.

In a similar fashion the dynamical invariants of the parametric oscillator (2.44) are given by the expression (4.19) with $c_{0} \neq 0$. In the Pinney solution (4.22) one can choose

$$
\begin{aligned}
& u=\frac{\omega \cos (\omega t) \cosh (\lambda t)-\lambda \sin (\omega t) \sinh (\lambda t)}{\cosh (\lambda t)}, \\
& v=\frac{\omega \sin (\omega t) \cosh (\lambda t)+\lambda \cos (\omega t) \sinh (\lambda t)}{\cosh (\lambda t)}
\end{aligned}
$$

as two linearly independent solutions of the classical equation of motion (2.45) with $W(u, v)=$ $\omega\left(\omega^{2}+\lambda^{2}\right)$. If $A=C$ and $B=0$, then

$$
\kappa=\left(\omega^{2}+\lambda^{2} \tanh ^{2}(\lambda t)\right)^{1 / 2}
$$

is a particular solution of the corresponding Ermakov equation:

$$
\kappa^{\prime \prime}+\left(\omega^{2}+\frac{2 \lambda^{2}}{\cosh ^{2}(\lambda t)}\right) \kappa=\frac{\omega^{2}\left(\lambda^{2}+\omega^{2}\right)^{2}}{\kappa^{3}} .
$$

The simplest positive energy integral for our parametric oscillator (2.44) is given by

$$
\begin{aligned}
E= & \left(\omega^{2}+\lambda^{2} \tanh ^{2}(\lambda t)\right) p^{2}+\lambda^{3} \frac{\sinh (\lambda t)}{\cosh ^{3}(\lambda t)}(p x+x p) \\
& +\frac{\lambda^{6} \sinh ^{2}(\lambda t)+\omega^{2}\left(\lambda^{2}+\omega^{2}\right)^{2} \cosh ^{6}(\lambda t)}{\cosh ^{6}(\lambda t)\left(\omega^{2}+\lambda^{2} \tanh ^{2}(\lambda t)\right)} x^{2} .
\end{aligned}
$$

Another possibility is to take a general solution of (2.45) with $c_{0}=0$.

4.3. An Extension to General Quadratic Hamiltonians. We consider the following generalization of the Lewis-Riesenfeld invariant (4.19) (see also [84], [150]).

Theorem 1. The dynamical invariants for the general quadratic Hamiltonian (3.7) are given by

$$
E=\frac{1}{\mu_{1}}\left(\kappa p-\frac{1}{2 a} \frac{d \kappa}{d t} x\right)^{2}+\frac{C_{0}}{\mu_{2} \kappa^{2}} x^{2}
$$

where $C_{0}$ is a constant,

$$
\mu_{1}=\exp \left(-\int_{0}^{t}(3 c+d) d s\right), \quad \mu_{2}=\exp \left(\int_{0}^{t}(c+3 d) d s\right),
$$

and $\kappa$ satisfies the auxiliary nonlinear equation:

$$
k \frac{d}{d t}\left(k \frac{d \kappa}{d t}\right)+4 a b k^{2} \kappa=\frac{C_{0}}{\kappa^{3}}
$$


where

$$
k=\frac{1}{2 a} \exp \left(2 \int_{0}^{t}(c+d) d s\right) .
$$

(For the self-adjoint Hamiltonians $c=d$.)

The case, $a=1 / 2, b=\omega^{2}(t) / 2$ and $c=d=0$, corresponds to the original invariant (4.19).

Proof. By Lemma 2 in order to find quadratic invariants of the form

$$
E=A p^{2}+B x^{2}+C p x+D x p
$$

we have to solve the following system of ordinary differential equations:

$$
\begin{aligned}
& \frac{d A}{d t}+2 a(C+D)-(3 c+d) A=0 \\
& \frac{d B}{d t}-2 b(C+D)+(c+3 d) B=0 \\
& \frac{d C}{d t}+2(a B-b A)-(c-d) C=0 \\
& \frac{d D}{d t}+2(a B-b A)-(c-d) D=0
\end{aligned}
$$

say, for arbitrary analytic coefficients $a(t), b(t), c(t)$ and $d(t)$. The substitution $C=C_{1}+D_{1}$, $D=C_{1}-D_{1}$ allows one to transform the last two equations:

$$
\begin{aligned}
& \frac{d C_{1}}{d t}+2(a B-b A)-(c-d) C_{1}=0 \\
& \frac{d D_{1}}{d t}=(c-d) D_{1}, \quad D_{1}=\text { constant } \exp \left(\int_{0}^{t}(c-d) d s\right) .
\end{aligned}
$$

Then

$$
C p x+D x p=C_{1}(p x+x p)+D_{1}(p x-x p)
$$

and, in view of the canonical commutation relation, the coefficient $D_{1}$ can be eliminated from the consideration as belonging to the linear invariants (see appendix $\mathrm{C}$ ).

Introducing integrating factors into (4.36), (4.37) and (4.40), we get

$$
\begin{array}{ll}
\frac{d}{d t}\left(\mu_{1} A\right)+4 a \mu_{1} C_{1}=0, & \frac{\mu_{1}^{\prime}}{\mu_{1}}=-3 c-d, \\
\frac{d}{d t}\left(\mu_{2} B\right)-4 b \mu_{2} C_{1}=0, & \frac{\mu_{2}^{\prime}}{\mu_{2}}=c+3 d, \\
\frac{d}{d t}\left(\mu_{3} C_{1}\right)+2 \mu_{3}(a B-b A)=0, & \frac{\mu_{3}^{\prime}}{\mu_{3}}=-c+d
\end{array}
$$

with $\mu_{3}^{2}=\mu_{1} \mu_{2}$. After the substitution

$$
\widetilde{A}=\mu_{1} A, \quad \widetilde{B}=\mu_{2} B, \quad \widetilde{C}=\mu_{3} C_{1},
$$

the system takes the form

$$
\frac{d \widetilde{A}}{d t}+4 a \sqrt{\frac{\mu_{1}}{\mu_{2}}} \widetilde{C}=0
$$




$$
\begin{aligned}
& \frac{d \widetilde{B}}{d t}-4 b \sqrt{\frac{\mu_{2}}{\mu_{1}}} \widetilde{C}=0 \\
& \frac{d \widetilde{C}}{d t}+2\left(a \sqrt{\frac{\mu_{1}}{\mu_{2}}} \widetilde{B}-b \sqrt{\frac{\mu_{2}}{\mu_{1}}} \widetilde{A}\right)=0
\end{aligned}
$$

Introducing a "proper time":

$$
\tau=\int_{0}^{t} 2 a \sqrt{\frac{\mu_{1}}{\mu_{2}}} d s
$$

we finally obtain:

$$
\begin{aligned}
& \frac{d \widetilde{A}}{d \tau}+2 \widetilde{C}=0 \\
& \frac{d \widetilde{B}}{d \tau}-2 \omega^{2}(\tau) \widetilde{C}=0 \\
& \frac{d \widetilde{C}}{d \tau}+\widetilde{B}-\omega^{2}(\tau) \widetilde{A}=0, \quad \omega^{2}(\tau)=\frac{b \mu_{2}}{a \mu_{1}},
\end{aligned}
$$

which is identical to the original Lewis-Riesenfeld system (4.13)-(4.15) (positivity of $\omega^{2}$ is not required). The solution is given by

$$
\widetilde{A}=\kappa^{2}, \quad \widetilde{B}=\left(\frac{d \kappa}{d \tau}\right)^{2}+\frac{C_{0}}{\kappa^{2}}, \quad \widetilde{C}=-\kappa \frac{d \kappa}{d \tau},
$$

where $\kappa$ satisfies the Ermakov equation:

$$
\frac{d^{2} \kappa}{d \tau^{2}}+\omega^{2}(\tau) \kappa=\frac{C_{0}}{\kappa^{3}}, \quad \omega^{2}(\tau)=\frac{b \mu_{2}}{a \mu_{1}},
$$

with respect to the new time (4.49). In view of

$$
\frac{d}{d \tau}=k \frac{d}{d t}, \quad k=\frac{1}{2 a} \exp \left(2 \int_{0}^{t}(c+d) d s\right)
$$

the Ermakov equation (4.54) is transformed into our auxiliary equation (4.33). A back substitution results in the dynamical invariant (4.31) when the square is completed.

Lemma 3. The dynamical invariant (4.31) can be represented in more symmetric form

$$
\begin{aligned}
E= & \left(\left(\mu p-\frac{1}{2 a}\left(\frac{d \mu}{d t}-(c+d) \mu\right) x\right)^{2}+\frac{C_{0}}{\mu^{2}} x^{2}\right) \\
& \times \exp \left(\int_{0}^{t}(c-d) d s\right),
\end{aligned}
$$

where $C_{0}$ is a constant and $\mu$ is a solution of the following auxiliary equation:

$$
\mu^{\prime \prime}-\frac{a^{\prime}}{a} \mu^{\prime}+\left(4 a b+\left(\frac{a^{\prime}}{a}-c-d\right)(c+d)-c^{\prime}-d^{\prime}\right) \mu=C_{0} \frac{(2 a)^{2}}{\mu^{3}} .
$$


Proof. Use the substitution

$$
\kappa=\mu \exp \left(-\int_{0}^{t}(c+d) d s\right)
$$

in (4.31) and (4.33). A somewhat different proof is given in [138].

The corresponding classical invariant is discussed, for example, in Refs. [141] and [150]. (Compare also our expression (4.56) with the one given in the last paper for the self-adjoint case; we give a detailed proof for the non-self-adjoint Hamiltonians and emphasize connection with the Ermakov equation.)

It is worth noting, in conclusion, that, if $\mu_{1}$ and $\mu_{2}$ are two linearly independent solutions of the linear equation:

$$
\mu^{\prime \prime}-\frac{a^{\prime}}{a} \mu^{\prime}+\left(4 a b+\left(\frac{a^{\prime}}{a}-c-d\right)(c+d)-c^{\prime}-d^{\prime}\right) \mu=0,
$$

the general solution of the nonlinear auxiliary equation (4.57) is given by

$$
\mu=\left(A \mu_{1}^{2}+2 B \mu_{1} \mu_{2}+C \mu_{2}^{2}\right)^{1 / 2},
$$

where the constants $A, B$ and $C$ are related according to

$$
A C-B^{2}=C_{0} \frac{(2 a)^{2}}{W^{2}\left(\mu_{1}, \mu_{2}\right)}
$$

with $W\left(\mu_{1}, \mu_{2}\right)=\mu_{1} \mu_{2}^{\prime}-\mu_{1}^{\prime} \mu_{2}=$ constant $(2 a)$ being the Wronskian of the two linearly independent solutions. This is a simple extension of Pinney's solution (4.22); our equations (4.57) and (4.59) form the generalized Ermakov system [43], [114]. Further generalization of the superposition formula (4.60)-(4.61) is discussed in Ref. [138]. (If $C_{0} \neq 0$, the substitution $\mu \rightarrow C_{0}^{1 / 4} \mu$ reduces equation (4.57) to a similar form with $C_{0}=1$.) Special case of the time-dependent damped harmonic oscillator, when $a=e^{-F(t)} / 2, b=\omega^{2}(t) e^{F(t)} / 2, F(t)=\int_{0}^{t} f(s) d s$ and $c=d=0$, is discussed in [82], [83].

4.4. An Example. The simplest energy operators have been already discussed in section 4.1 for all models of quantum oscillators under consideration. In order to demonstrate how the general approach works we discuss the united Hamiltonian (2.11), when $a=\left(\omega_{0} / 2\right) e^{-2 \lambda t}, b=\left(\omega_{0} / 2\right) e^{2 \lambda t}$ and $c=0, d=-\mu$. A direct calculation shows that the function

$$
\kappa=\sqrt{\frac{\omega_{0}}{2}} e^{-\lambda t}
$$

satisfies the following equation

$$
\kappa^{\prime \prime}+2 \lambda \kappa^{\prime}+\omega_{0}^{2} \kappa=\left(\frac{\omega_{0} \omega}{2}\right)^{2} \frac{e^{-4 \lambda t}}{\kappa^{3}}, \quad \omega^{2}=\omega_{0}^{2}-(\lambda-\mu)^{2}>0,
$$

which corresponds to the nonlinear auxiliary equation (4.57) with $C_{0}=\omega^{2} / 4$. The quadratic invariant (4.56) simplifies to the previously found expression (4.12). Solution (4.60) can be used for the most general case. Details are left to the reader. 
4.5. Factorization of the Dynamical Invariant. Following Ref. [27] the energy operator (4.56) can be presented in the standard harmonic oscillator form:

$$
E=\frac{\omega(t)}{2}\left(\widehat{a}(t) \widehat{a}^{\dagger}(t)+\widehat{a}^{\dagger}(t) \widehat{a}(t)\right)
$$

where

$$
\begin{gathered}
\omega(t)=\omega_{0} \exp \left(\int_{0}^{t}(c-d) d s\right), \quad \omega_{0}=2 \sqrt{C_{0}}>0, \\
\widehat{a}(t)=\left(\frac{\sqrt{\omega_{0}}}{2 \mu}-i \frac{\mu^{\prime}-(c+d) \mu}{2 a \sqrt{\omega_{0}}}\right) x+\frac{\mu}{\sqrt{\omega_{0}}} \frac{\partial}{\partial x} \\
\widehat{a}^{\dagger}(t)=\left(\frac{\sqrt{\omega_{0}}}{2 \mu}+i \frac{\mu^{\prime}-(c+d) \mu}{2 a \sqrt{\omega_{0}}}\right) x-\frac{\mu}{\sqrt{\omega_{0}}} \frac{\partial}{\partial x}
\end{gathered}
$$

and $\mu$ is a solution of the nonlinear auxiliary equation (4.57). Here the time-dependent annihilation $\widehat{a}(t)$ and creation $\widehat{a}^{\dagger}(t)$ operators satisfy the usual commutation relation:

$$
\widehat{a}(t) \widehat{a}^{\dagger}(t)-\widehat{a}^{\dagger}(t) \widehat{a}(t)=1 \text {. }
$$

The oscillator-type spectrum and the corresponding time-dependent eigenfunctions of the dynamical invariant $E$ can be obtain now in a standard way by using the Heisenberg-Weyl algebra of the rasing and lowering operators (a "second quantization" [91], the Fock states). Explicit solution of the Cauchy initial value problem in terms of the quadratic invariant eigenfunction expansion is found in Ref. [138. In addition the $n$-dimensional oscillator wave functions form a basis of the irreducible unitary representation of the Lie algebra of the noncompact group $S U(1,1)$ corresponding to the discrete positive series $\mathcal{D}_{+}^{j}$ (see [101], [111] and [132]). Our operators (4.66)-(4.67) allow one to extend these group-theoretical properties to the general dynamical invariant (4.64). We shall further elaborate on these connections elsewhere.

\section{Application to the Cauchy initial Value Problems}

Explicit solution of the initial value problem in terms of eigenfunctions of the general quadratic invariant is given in Ref. [138. Here we formulate the following uniqueness result.

Lemma 4. Suppose that the expectation value

$$
\left\langle H_{0}\right\rangle=\left\langle\psi, H_{0} \psi\right\rangle \geq 0
$$

for a positive quadratic operator

$$
H_{0}=f(t)(\alpha(t) p+\beta(t) x)^{2}+g(t) x^{2} \quad(f(t) \geq 0, g(t)>0)
$$

$(\alpha(t)$ and $\beta(t)$ are real-valued functions) vanishes for all $t \in[0, T)$ :

$$
\left\langle H_{0}\right\rangle=\left\langle H_{0}\right\rangle(t)=\left\langle H_{0}\right\rangle(0)=0,
$$

when $\psi(x, 0)=0$ almost everywhere. Then the corresponding Cauchy initial value problem

$$
i \frac{\partial \psi}{\partial t}=H \psi, \quad \psi(x, 0)=\varphi(x)
$$

may have only one solution, when $x \psi(x, t) \in L^{2}(\mathbb{R})$ (if $H_{0}=g(t) I$, where $I=i d$ is the identity operator, $\psi \in L^{2}(\mathbb{R})$ ). 
Here it is not assumed that $H_{0}$ is the quantum integral of motion when $\frac{d}{d t}\left\langle H_{0}\right\rangle \equiv 0$.

Proof. If there are two solutions:

$$
i \frac{\partial \psi_{1}}{\partial t}=H \psi_{1}, \quad i \frac{\partial \psi_{2}}{\partial t}=H \psi_{2}
$$

with the same initial condition $\psi_{1}(x, 0)=\psi_{2}(x, 0)=\varphi(x)$, then by the superposition principle the function $\psi=\psi_{1}-\psi_{2}$ is also a solution with respect to the zero initial data $\psi(x, 0)=\varphi(x)-\varphi(x)=$ 0 . By the hypothesis of the lemma

$$
\left\langle\psi, H_{0} \psi\right\rangle=f(t)\langle(\alpha p+\beta x) \psi,(\alpha p+\beta x) \psi\rangle+g(t)\langle x \psi, x \psi\rangle=0
$$

for all $t \in[0, T)$. Therefore $x \psi(x, t)=x\left(\psi_{1}(x, t)-\psi_{2}(x, t)\right)=0$ and $\psi_{1}(x, t)=\psi_{2}(x, t)$ almost everywhere for all $t>0$ by the axiom of the inner product in $L^{2}(\mathbb{R})$.

In order to apply this lemma to the variable Hamiltonians one has to identify the corresponding positive operators $H_{0}$ and establish their required uniqueness dynamics properties with respect to the zero initial data. In addition to the simplest available dynamical invariant (B.1), it is worth exploring other (quadratic) possibilities. The authors believe that it is interesting and may be important on its own. For example, our approach gives an opportunity to determine a complete time-evolution of the standard deviations (B.9)-(B.10) for each of the generalized harmonic oscillators under consideration. The details will be discussed elsewhere.

5.1. The Caldirola-Kanai Hamiltonian. The required operators are given by

$$
\begin{gathered}
H=H_{0}=\frac{\omega_{0}}{2}\left(e^{-2 \lambda t} p^{2}+e^{2 \lambda t} x^{2}\right), \\
L=\frac{\partial H}{\partial t}=\lambda \omega_{0}\left(-e^{-2 \lambda t} p^{2}+e^{2 \lambda t} x^{2}\right), \\
E=\frac{\omega_{0}}{2}\left(e^{-2 \lambda t} p^{2}+e^{2 \lambda t} x^{2}\right)+\frac{\lambda}{2}(p x+x p), \quad \frac{d}{d t}\langle E\rangle=0 .
\end{gathered}
$$

By 3.5

$$
\frac{d}{d t}\langle H\rangle=\left\langle\frac{\partial H}{\partial t}\right\rangle=\langle L\rangle
$$

Applying formula (3.4) one gets

$$
\begin{aligned}
\frac{d}{d t}\langle L\rangle= & 2 \lambda^{2} \omega_{0}\left(e^{-2 \lambda t}\left\langle p^{2}\right\rangle+e^{2 \lambda t}\left\langle x^{2}\right\rangle\right) \\
& +2 \lambda \omega_{0}^{2}\langle p x+x p\rangle
\end{aligned}
$$

and

with the help of (5.5) and (5.7).

$$
\frac{d}{d t}\langle L\rangle+4 \omega^{2}\langle H\rangle=4 \omega_{0}^{2}\langle E\rangle_{0}
$$

In view of (5.8) and (5.10) the dynamics of the Hamiltonian expectation value $\langle H\rangle$ is governed by the following second-order differential equation

$$
\frac{d^{2}}{d t^{2}}\langle H\rangle+4 \omega^{2}\langle H\rangle=4 \omega_{0}^{2}\langle E\rangle_{0}
$$


with the unique solution given by

$$
\langle H\rangle=\frac{\omega^{2}\langle H\rangle_{0}-\omega_{0}^{2}\langle E\rangle_{0}}{\omega^{2}} \cos (2 \omega t)+\frac{1}{2 \omega}\left\langle\frac{\partial H}{\partial t}\right\rangle_{0} \sin (2 \omega t)+\frac{\omega_{0}^{2}}{\omega^{2}}\langle E\rangle_{0} .
$$

The hypotheses of Lemma 4 are satisfied. Our solution allows to determine a complete timeevolution of the expectation values of the operators $p^{2}, x^{2}$ and $p x+x p$. Further details are left to the reader.

5.2. The Modified Caldirola-Kanai Hamiltonian. The required operators are

$$
\begin{gathered}
H=\frac{\omega_{0}}{2}\left(e^{-2 \lambda t} p^{2}+e^{2 \lambda t} x^{2}\right)-\lambda(p x+x p), \\
L=\frac{\partial H}{\partial t}=\lambda \omega_{0}\left(-e^{-2 \lambda t} p^{2}+e^{2 \lambda t} x^{2}\right)=\frac{\partial H_{0}}{\partial t}, \\
E=\frac{\omega_{0}}{2}\left(e^{-2 \lambda t} p^{2}+e^{2 \lambda t} x^{2}\right)-\frac{\lambda}{2}(p x+x p) .
\end{gathered}
$$

We consider the expectation value $\left\langle H_{0}\right\rangle$ of the positive operator

$$
H_{0}=\frac{\omega_{0}}{2}\left(e^{-2 \lambda t} p^{2}+e^{2 \lambda t} x^{2}\right) \text {. }
$$

In this case $H=2 E-H_{0}$, and

$$
\begin{aligned}
\frac{d}{d t}\langle H\rangle & =\left\langle\frac{\partial H}{\partial t}\right\rangle=\langle L\rangle=-\frac{d}{d t}\left\langle H_{0}\right\rangle, \\
\frac{d}{d t}\langle L\rangle & =4 \omega^{2}\left\langle H_{0}\right\rangle-4 \omega_{0}^{2}\langle E\rangle_{0},
\end{aligned}
$$

which results in the differential equation (5.11) with the explicit solution

$$
\left\langle H_{0}\right\rangle=\frac{\omega^{2}\left\langle H_{0}\right\rangle_{0}-\omega_{0}^{2}\langle E\rangle_{0}}{\omega^{2}} \cos (2 \omega t)-\frac{1}{2 \omega}\left\langle\frac{\partial H_{0}}{\partial t}\right\rangle_{0} \sin (2 \omega t)+\frac{\omega_{0}^{2}}{\omega^{2}}\langle E\rangle_{0}
$$

of the initial value problem. The hypotheses of the lemma are satisfied.

5.3. The United Model. The related operators can be conveniently extended as follows

$$
\begin{gathered}
H_{0}=\frac{\omega_{0}}{2} e^{\mu t}\left(e^{-2 \lambda t} p^{2}+e^{2 \lambda t} x^{2}\right), \\
L=e^{\mu t}\left(-e^{-2 \lambda t} p^{2}+e^{2 \lambda t} x^{2}\right), \\
M=e^{\mu t}(p x+x p)
\end{gathered}
$$

and

$$
\begin{aligned}
E & =H_{0}(t)+\frac{1}{2}(\lambda-\mu) M(t) \\
& =\frac{\omega_{0}}{2} e^{\mu t}\left(e^{-2 \lambda t} p^{2}+e^{2 \lambda t} x^{2}\right)+\frac{1}{2}(\lambda-\mu) e^{\mu t}(p x+x p) .
\end{aligned}
$$

Then by Lemma 2

$$
\begin{gathered}
\frac{d}{d t}\langle M\rangle=-2 \omega_{0}\langle L\rangle, \\
\frac{d}{d t}\left\langle H_{0}\right\rangle=\omega_{0}(\lambda-\mu)\langle L\rangle,
\end{gathered}
$$




$$
\frac{d}{d t}\langle E\rangle=0
$$

and

$$
\frac{d}{d t}\langle L\rangle=4 \frac{\lambda-\mu}{\omega_{0}}\left\langle H_{0}\right\rangle+2 \omega_{0}\langle M\rangle .
$$

In terms of the energy operator

$$
\frac{d}{d t}\langle L\rangle+\frac{4 \omega^{2}}{(\lambda-\mu) \omega_{0}}\left\langle H_{0}\right\rangle=\frac{4 \omega_{0}}{\lambda-\mu}\langle E\rangle
$$

and as a result

$$
\frac{d^{2}}{d t^{2}}\left\langle H_{0}\right\rangle+4 \omega^{2}\left\langle H_{0}\right\rangle=4 \omega_{0}^{2}\langle E\rangle_{0}, \quad \omega=\sqrt{\omega_{0}^{2}-(\lambda-\mu)^{2}}>0
$$

with the unique solution of the initial value problem given by

$$
\begin{aligned}
\left\langle H_{0}\right\rangle= & \frac{\omega^{2}\left\langle H_{0}\right\rangle_{0}-\omega_{0}^{2}\langle E\rangle_{0}}{\omega^{2}} \cos (2 \omega t) \\
& +\frac{1}{2}(\lambda-\mu) \frac{\omega_{0}}{\omega}\langle L\rangle_{0} \sin (2 \omega t)+\frac{\omega_{0}^{2}}{\omega^{2}}\langle E\rangle_{0} .
\end{aligned}
$$

The hypotheses of Lemma 4 are satisfied.

5.4. The Modified Oscillator. The required operators are

$$
\begin{aligned}
H & =(\cos t p+\sin t x)^{2} \\
& =\cos ^{2} t p^{2}+\sin ^{2} t x^{2}+\sin t \cos t(p x+x p) \\
& =\frac{1}{2}\left(p^{2}+x^{2}\right)+\frac{1}{2} \cos 2 t\left(p^{2}-x^{2}\right)+\frac{1}{2} \sin 2 t(p x+p x) \\
& =H_{0}+E(t),
\end{aligned}
$$

where

and

$$
\begin{gathered}
H_{0}=\frac{1}{2}\left(p^{2}+x^{2}\right), \\
E=E(t)=\frac{1}{2} \cos 2 t\left(p^{2}-x^{2}\right)+\frac{1}{2} \sin 2 t(p x+p x)
\end{gathered}
$$

Here

$$
L=\frac{\partial H}{\partial t}=\frac{\partial E}{\partial t}=-\sin 2 t\left(p^{2}-x^{2}\right)+\cos 2 t(p x+p x) .
$$

$$
\frac{d}{d t}\left\langle H_{0}\right\rangle=\frac{d}{d t}\langle H\rangle=\left\langle\frac{\partial H}{\partial t}\right\rangle=\left\langle\frac{\partial E}{\partial t}\right\rangle=\langle L\rangle
$$

and

$$
\frac{d}{d t}\langle L\rangle=4\left\langle H_{0}\right\rangle \text {. }
$$

The expectation value $\left\langle H_{0}\right\rangle$ satisfies the following differential equation

with the explicit solution

$$
\frac{d^{2}}{d t^{2}}\left\langle H_{0}\right\rangle=4\left\langle H_{0}\right\rangle
$$

$$
\left\langle H_{0}\right\rangle=\left\langle H_{0}\right\rangle_{0} \cosh (2 t)+\frac{1}{2}\langle L\rangle_{0} \sinh (2 t) .
$$


The hypotheses of Lemma 4 are satisfied.

5.5. The Modified Damped Oscillator. Let $\hbar=m \omega_{0}=1$ in the Hamiltonian (2.21):

$$
H=\frac{\omega_{0}}{2}\left(\frac{p^{2}}{\cosh ^{2}(\lambda t)}+\cosh ^{2}(\lambda t) x^{2}\right)
$$

without loss of generality. The corresponding energy operator can be found as follows

$$
\begin{aligned}
E= & \frac{\omega_{0}}{2 \cosh ^{2}(\lambda t)} p^{2}+\frac{\omega_{0}^{2} \sinh ^{2}(\lambda t)+\omega^{2}}{2 \omega_{0}} x^{2} \\
& +\frac{\lambda}{2} \tanh (\lambda t)(p x+x p), \quad \frac{d}{d t}\langle E\rangle=0,
\end{aligned}
$$

in view of (4.5)-(4.7) (one should replace $A \leftrightarrow B, C \rightarrow-C$ in the momentum representation).

Introducing the following complementary operators

$$
\begin{aligned}
H_{0} & =\frac{p^{2}}{\cosh ^{2}(\lambda t)}+\cosh ^{2}(\lambda t) x^{2}, \\
L & =\frac{p^{2}}{\cosh ^{2}(\lambda t)}-\cosh ^{2}(\lambda t) x^{2}, \\
M & =p x+x p
\end{aligned}
$$

we get

$$
\begin{aligned}
\frac{d}{d t}\left\langle H_{0}\right\rangle & =-2 \lambda \tanh (\lambda t)\langle L\rangle, \\
\frac{d}{d t}\langle L\rangle & =-2 \lambda \tanh (\lambda t)\left\langle H_{0}\right\rangle-2 \omega_{0}\langle M\rangle, \\
\frac{d}{d t}\langle M\rangle & =2 \omega_{0}\langle L\rangle .
\end{aligned}
$$

Then

$$
\begin{aligned}
E= & \frac{\omega_{0}}{2}\left(1-\frac{\lambda^{2}}{2 \omega_{0}^{2} \cosh ^{2}(\lambda t)}\right) H_{0}+\frac{\lambda^{2}}{4 \omega_{0} \cosh ^{2}(\lambda t)} L \\
& +\frac{\lambda}{2} \tanh (\lambda t) M
\end{aligned}
$$

and, eliminating $\langle M\rangle$ and $\langle L\rangle$ from the system, one gets:

$$
\frac{d^{2}}{d t^{2}}\left\langle H_{0}\right\rangle-\frac{4 \lambda}{\sinh (2 \lambda t)} \frac{d}{d t}\left\langle H_{0}\right\rangle+2\left(2 \omega^{2}+\frac{\lambda^{2}}{\cosh ^{2}(\lambda t)}\right)\left\langle H_{0}\right\rangle=8 \omega_{0}\langle E\rangle_{0} .
$$

The required initial conditions:

$$
\left(\frac{d}{d t}\left\langle H_{0}\right\rangle\right)_{0}=0, \quad\left(\operatorname{coth}(\lambda t) \frac{d}{d t}\left\langle H_{0}\right\rangle\right)_{0}=-2 \lambda\langle L\rangle_{0}
$$

follow from (5.44). The unique explicit solution is given by

$$
\left\langle H_{0}\right\rangle=-\lambda \frac{\lambda^{2}\langle E\rangle_{0}+\omega_{0} \omega^{2}\langle L\rangle_{0}}{\omega_{0} \omega^{2}\left(2 \omega^{2}+\lambda^{2}\right)}
$$




$$
\begin{aligned}
& \times\left(2 \omega \tanh (\lambda t) \sin (2 \omega t)+\lambda\left(1+\tanh ^{2}(\lambda t)\right) \cos (2 \omega t)\right) \\
& +2\langle E\rangle_{0} \frac{\omega_{0}}{\omega^{2}}\left(1-\frac{\lambda^{2}}{2 \omega_{0}^{2} \cosh ^{2}(\lambda t)}\right)
\end{aligned}
$$

(see appendix D). The hypotheses of Lemma 4 are satisfied.

5.6. The Modified Parametric Oscillator. In the case (2.37), the energy operator (4.11) is a positive operator:

$$
\langle E\rangle=\tanh ^{2}(\lambda t+\delta)\left\langle p^{2}\right\rangle+\operatorname{coth}^{2}(\lambda t+\delta)\left\langle x^{2}\right\rangle=\langle E\rangle_{0}>0 .
$$

The related operators are

$$
\begin{aligned}
L & =\tanh ^{2}(\lambda t+\delta) p^{2}-\operatorname{coth}^{2}(\lambda t+\delta) x^{2}, \\
M & =p x+x p \\
H & =\frac{\omega}{2} E+\frac{\lambda}{\sinh (2 \lambda t+2 \delta)} M
\end{aligned}
$$

with

From here

$$
\frac{d}{d t}\langle L\rangle=-2 \omega\langle M\rangle, \quad \frac{d}{d t}\langle M\rangle=-2 \omega\langle L\rangle
$$

$$
\frac{d^{2}}{d t^{2}}\langle L\rangle+4 \omega^{2}\langle L\rangle=0, \quad \frac{d^{2}}{d t^{2}}\langle M\rangle+4 \omega^{2}\langle M\rangle=0,
$$

which determines the time-evolution of the expectation values.

5.7. Parametric Oscillators. In general the Lewis-Riesenfeld quadratic invariant (4.19) for the parametric oscillator (2.50) is obviously a positive operator for real-valued solutions of the Ermakov equation (4.17) that satisfies the conditions of our lemma.

5.8. General Quadratic Hamiltonian. In the case of Hamiltonian (3.7) applying formula (3.9) to the operators, $O=\left\{p^{2}, x^{2}, p x+x p\right\}$, one obtains [27]:

$$
\frac{d}{d t}\left(\begin{array}{c}
\left\langle p^{2}\right\rangle \\
\left\langle x^{2}\right\rangle \\
\langle p x+x p\rangle
\end{array}\right)=\left(\begin{array}{ccc}
-3 c(t)-d(t) & 0 & -2 b(t) \\
0 & c(t)+3 d(t) & 2 a(t) \\
4 a(t) & -4 b(t) & -c(t)+d(t)
\end{array}\right)\left(\begin{array}{c}
\left\langle p^{2}\right\rangle \\
\left\langle x^{2}\right\rangle \\
\langle p x+x p\rangle
\end{array}\right) .
$$

This system has a unique solution for suitable coefficients [59], which allows one to apply Lemma 4, say, for the positive operator $x^{2}$. Our Theorem 1 provides another choice of positive operators. On the second thought a positive integral (B.3) determines time-evolution of the squared norm and guarantees uniqueness in $L^{2}(\mathbb{R})$. Details are left to the reader.

Acknowledgments. We thank Professor Carlos Castillo-Chávez, Professor Victor V. Dodonov, Professor Vladimir I. Man'ko and Professor Kurt Bernardo Wolf for support, valuable discussions and encouragement. The authors are indebted to Professor George A. Hagedorn for kindly pointing out the papers [55] and [56] to our attention. We thank David Murillo for help. The authors are grateful to Professor Peter G. L. Leach for careful reading of the manuscript - his numerous suggestions have helped to improve the presentation. One of the authors (RCS) is supported by the following National Science Foundation programs: Louis Stokes Alliances for Minority Participation (LSAMP): NSF Cooperative Agreement No. HRD-0602425 (WAESO LSAMP Phase IV); Alliances 
for Graduate Education and the Professoriate (AGEP): NSF Cooperative Agreement No. HRD0450137 (MGE@MSA AGEP Phase II).

\section{Appendix A. The Ehrenfest Theorems}

Application of formula (3.5) to the position $x$ and momentum $p$ operators allows one to derive the Ehrenfest theorem [42], [102], [129] for the models of oscillators under consideration.

For the Caldirola-Kanai Hamiltonian (2.1) one gets

$$
\frac{d}{d t}\langle x\rangle=\omega_{0} e^{-2 \lambda t}\langle p\rangle, \quad \frac{d}{d t}\langle p\rangle=-\omega_{0} e^{2 \lambda t}\langle x\rangle .
$$

Elimination of the expectation value $\langle p\rangle$ from this system results in the classical equation of motion for a damped oscillator [5], [79]:

$$
\frac{d^{2}}{d t^{2}}\langle x\rangle+2 \lambda \frac{d}{d t}\langle x\rangle+\omega_{0}^{2}\langle x\rangle=0
$$

For the modified Caldirola-Kanai Hamiltonian (2.6) the system

$$
\frac{d}{d t}\langle x\rangle=\omega_{0} e^{-2 \lambda t}\langle p\rangle-2 \lambda\langle x\rangle, \quad \frac{d}{d t}\langle p\rangle=-\omega_{0} e^{2 \lambda t}\langle x\rangle+2 \lambda\langle p\rangle
$$

gives the same classical equation.

In the case of the united model (2.11) one should use the differentiation formula (3.10). Then

$$
\frac{d}{d t}\langle x\rangle=\omega_{0} e^{-2 \lambda t}\langle p\rangle-2 \mu\langle x\rangle, \quad \frac{d}{d t}\langle p\rangle=-\omega_{0} e^{2 \lambda t}\langle x\rangle
$$

and the second order equations are given by

The general solutions are

$$
\begin{gathered}
\frac{d^{2}}{d t^{2}}\langle x\rangle+2(\lambda+\mu) \frac{d}{d t}\langle x\rangle+\left(\omega_{0}^{2}+4 \lambda \mu\right)\langle x\rangle=0, \\
\frac{d^{2}}{d t^{2}}\langle p\rangle+2(\mu-\lambda) \frac{d}{d t}\langle p\rangle+\omega_{0}^{2}\langle p\rangle=0 .
\end{gathered}
$$

$$
\begin{aligned}
& \langle x\rangle=A e^{-(\lambda+\mu) t} \sin (\omega t+\delta), \\
& \langle p\rangle=B e^{(\lambda-\mu) t} \sin (\omega t+\gamma)
\end{aligned}
$$

where $\omega=\sqrt{\omega_{0}^{2}-(\lambda-\mu)^{2}}>0$.

In a similar fashion for a modified oscillator with the Hamiltonian (2.17) we obtain

$$
\begin{aligned}
\frac{d}{d t}\langle x\rangle & =2 \cos ^{2} t\langle p\rangle+2 \sin t \cos t\langle x\rangle, \\
\frac{d}{d t}\langle p\rangle & =-2 \sin ^{2} t\langle x\rangle-2 \sin t \cos t\langle p\rangle .
\end{aligned}
$$

Then

$$
\frac{d^{2}}{d t^{2}}\langle x\rangle+2 \tan t \frac{d}{d t}\langle x\rangle-2\langle x\rangle=0
$$

which coincides with the characteristic equation (1.6) in this case [28]. 
In the case of the damped oscillator of Chruściński and Jurkowski one obtains

$$
\begin{aligned}
\frac{d}{d t}\langle x\rangle & =\frac{\langle p\rangle}{m \cosh ^{2}(\lambda t)}, \\
\frac{d}{d t}\langle p\rangle & =-m \omega_{0}^{2} \cosh ^{2}(\lambda t)\langle x\rangle .
\end{aligned}
$$

The Ehrenfest theorems coincide with the Newtonian equations of motion [25]:

$$
\begin{aligned}
& \frac{d^{2}}{d t^{2}}\langle x\rangle+2 \lambda \tanh (\lambda t) \frac{d}{d t}\langle x\rangle+\omega_{0}^{2}\langle x\rangle=0 \\
& \frac{d^{2}}{d t^{2}}\langle p\rangle-2 \lambda \tanh (\lambda t) \frac{d}{d t}\langle p\rangle+\omega_{0}^{2}\langle p\rangle=0
\end{aligned}
$$

with the general solutions given by

$$
\begin{gathered}
\langle x\rangle=A \frac{\sin (\omega t+\delta)}{\cosh (\lambda t)}, \quad \omega=\sqrt{\omega_{0}^{2}-\lambda^{2}}>0, \\
\langle p\rangle=B(\lambda \cos (\omega t+\delta) \sinh (\lambda t)+\omega \sin (\omega t+\delta) \cosh (\lambda t)),
\end{gathered}
$$

respectively. It is worth noting that both equations (A.2) and (A.14) give the same frequency of oscillations for the damped motion; see [25] for more details.

Combining all models together for the general quadratic Hamiltonian (3.7):

$$
\frac{d}{d t}\langle x\rangle=2 a(t)\langle p\rangle+2 d(t)\langle x\rangle, \quad \frac{d}{d t}\langle p\rangle=-2 b(t)\langle x\rangle-2 c(t)\langle p\rangle
$$

with the help of (3.10). The Newtonian-type equation of motion for the expectation values has the form

$$
\frac{d^{2}}{d t^{2}}\langle x\rangle-\tau(t) \frac{d}{d t}\langle x\rangle+4 \sigma(t)\langle x\rangle=0
$$

with

$$
\tau(t)=\frac{a^{\prime}}{a}-2 c+2 d, \quad \sigma(t)=a b-c d+\frac{d}{2}\left(\frac{a^{\prime}}{a}-\frac{d^{\prime}}{d}\right) .
$$

In order to explain a connection with the characteristic equation (1.6)-(1.7) we temporarily replace $c \rightarrow c_{0}$ and $d \rightarrow d_{0}$ in the original Hamiltonian (1.1). Then it takes the standard form (3.7), if $c_{0}=c+d$ and $d_{0}=c$. Using the new notations in (1.6)-(1.7) we find

$$
\tau-\tau_{0}=4(d-c), \quad \sigma-\sigma_{0}=\frac{a}{2}\left(\frac{c-d}{a}\right)^{\prime} .
$$

Therefore our characteristic equation (1.6) coincides with the corresponding Ehrenfest theorem (A.19) only in the case of self-adjoint Hamiltonians, when $c=d$ (or $c_{0}=2 d_{0}$ ). The united model shows that these equations are different otherwise. 


\section{Appendix B. The Heisenberg Uncertainty Relation Revisited}

A detailed review with an extensive list of references is given in Refs. [38] and [109] (see also [99]). We only discuss the Heisenberg uncertainty relation for the position $x$ and momentum $p=-i \partial / \partial x$ operators (in the units of $\hbar$ ) in the case of the general quadratic Hamiltonian (3.7). By our Lemma 2 the simplest integral of motion is given by

$$
E_{0}=\exp \left(\int_{0}^{t}(c(\tau)-d(\tau)) d \tau\right)(p x-x p)
$$

with

$$
[x, p]=x p-p x=i
$$

This implies the following time evolution:

$$
\langle\psi, \psi\rangle=\exp \left(\int_{0}^{t}(d(\tau)-c(\tau)) d \tau\right)\langle\psi, \psi\rangle_{0}
$$

of the squared norm of the wave functions.

With the expectation values

$$
\bar{x}=\frac{\langle x\rangle}{\langle 1\rangle}=\frac{\langle\psi, x \psi\rangle}{\langle\psi, \psi\rangle}, \quad \bar{p}=\frac{\langle p\rangle}{\langle 1\rangle}=\frac{\langle\psi, p \psi\rangle}{\langle\psi, \psi\rangle}
$$

and the operators

$$
\Delta x=x-\bar{x}, \quad \Delta p=p-\bar{p}
$$

let us consider

$$
\begin{aligned}
0 & \leq\langle(\Delta x+i \lambda \Delta p) \psi,(\Delta x+i \lambda \Delta p) \psi\rangle \\
& =\langle\psi,(\Delta x-i \lambda \Delta p)(\Delta x+i \lambda \Delta p) \psi\rangle \\
& =\left\langle(\Delta x)^{2}\right\rangle-\lambda\langle 1\rangle+\lambda^{2}\left\langle(\Delta p)^{2}\right\rangle
\end{aligned}
$$

for a real parameter $\lambda$. Here we have used the operator identity

$$
(\Delta x-i \lambda \Delta p)(\Delta x+i \lambda \Delta p)=(\Delta x)^{2}-\lambda+\lambda^{2}(\Delta p)^{2} .
$$

Then one gets

$$
\left\langle(\Delta p)^{2}\right\rangle\left\langle(\Delta x)^{2}\right\rangle \geq \frac{1}{4}\langle 1\rangle^{2}=\frac{1}{4} \exp \left(2 \int_{0}^{t}(d(\tau)-c(\tau)) d \tau\right),
$$

if $\langle 1\rangle_{0}=\langle\psi, \psi\rangle_{0}=1$. For the standard deviations:

$$
\begin{aligned}
& (\delta p)^{2}=\frac{\left\langle(\Delta p)^{2}\right\rangle}{\langle 1\rangle}=\overline{\left(p^{2}\right)}-(\bar{p})^{2}, \\
& (\delta x)^{2}=\frac{\left\langle(\Delta x)^{2}\right\rangle}{\langle 1\rangle}=\overline{\left(x^{2}\right)}-(\bar{x})^{2},
\end{aligned}
$$

we finally obtain

$$
\delta p \delta x \geq \frac{1}{2}
$$

in the units of $\hbar$. It is worth noting that Eq. (B.11) is derived, in fact, for any operators $x$ and $p$ with the commutator (B.2) - the structure of the quadratic Hamiltonian (3.7) has only been 
used in the norm $(\underline{B .3})$. Time-evolution of the standard deviations $(\underline{B .9})-(\underline{B .10})$ will be discussed elsewhere.

\section{Appendix C. Linear Integrals of Motion: The \\ DODONOV-MALKIN-MAN'KO-TRIFONOV INVARIANTS}

All invariants of the form

$$
P=A(t) p+B(t) x+C(t)
$$

for the general quadratic Hamiltonian (3.7) can be found as follows (see, for example, [37, [39], [95], [98] and references therein). Use of the differentiation formula (3.10) results in the following system:

$$
\begin{aligned}
\frac{d A}{d t} & =2 c(t) A-2 a(t) B \\
\frac{d B}{d t} & =2 b(t) A-2 d(t) B \\
\frac{d C}{d t} & =(c(t)-d(t)) C .
\end{aligned}
$$

The last equation is explicitly integrated and elimination of $B$ and $A$ from (C.2) and (C.3), respectively, gives the second-order equations:

$$
\begin{aligned}
& A^{\prime \prime}-\left(\frac{a^{\prime}}{a}+2 c-2 d\right) A^{\prime}+4\left(a b-c d+\frac{c}{2}\left(\frac{a^{\prime}}{a}-\frac{c^{\prime}}{c}\right)\right) A=0 \\
& B^{\prime \prime}-\left(\frac{b^{\prime}}{b}+2 c-2 d\right) B^{\prime}+4\left(a b-c d-\frac{d}{2}\left(\frac{b^{\prime}}{b}-\frac{d^{\prime}}{d}\right)\right) B=0 .
\end{aligned}
$$

The first is equivalent to our characteristic equation (1.6)-(1.7) and coincides with the Ehrenfest theorem (A.19) - (A.20) when $c \leftrightarrow d$.

Thus the linear quantum invariants are given by

$$
P=A(t) p+\frac{2 c(t) A(t)-A^{\prime}(t)}{2 a(t)} x+C_{0} \exp \left(\int_{0}^{t}(c(\tau)-d(\tau)) d \tau\right),
$$

where $A(t)$ is a general solution of equation (C.5) depending upon two parameters and $C_{0}$ is the third constant. Our Theorem 1 gives a similar description of the quadratic invariants in terms of solutions of the auxiliary equation (4.33). Relations between linear and quadratic invariants are analyzed in [138]. Group-theoretical applications are discussed in [22], [36], [39], [95], [37], [91, [98] and elsewhere.

\section{Appendix D. An Elementary Differential Equation}

The nonhomogeneous differential equation of the form

$$
y^{\prime \prime}-\frac{4 \lambda}{\sinh (2 \lambda t+2 \gamma)} y^{\prime}+\left(\omega^{2}+\frac{2 \lambda^{2}}{\cosh ^{2}(\lambda t+\gamma)}\right) y=1
$$

( $\omega, \lambda$ and $\gamma$ are some parameters) has the following general solution:

$$
y=C_{1} y_{1}(t)+C_{2} y_{2}(t)+Y(t),
$$


where $C_{1}$ and $C_{2}$ are constants,

$$
\begin{aligned}
& y_{1}=\omega \tanh (\lambda t+\gamma) \cos (\omega t)-\lambda\left(1+\tanh ^{2}(\lambda t+\gamma)\right) \sin (\omega t), \\
& y_{2}=\omega \tanh (\lambda t+\gamma) \sin (\omega t)+\lambda\left(1+\tanh ^{2}(\lambda t+\gamma)\right) \cos (\omega t)
\end{aligned}
$$

are the fundamental solutions of the corresponding homogeneous equation with the Wronskian given by

$$
W\left(y_{1}, y_{2}\right)=\omega\left(\omega^{2}+4 \lambda^{2}\right) \tanh ^{2}(\lambda t+\gamma)
$$

and

$$
Y=\frac{1}{\omega^{2}}\left(1-\frac{2 \lambda^{2}}{\left(\omega^{2}+4 \lambda^{2}\right) \cosh ^{2}(\lambda t+\gamma)}\right)
$$

is a particular solution of the nonhomogeneous equation.

One can also verify that functions:

$$
\begin{aligned}
& z_{1}=\omega \cos (\omega t)-\lambda \operatorname{coth}(\lambda t+\gamma) \sin (\omega t), \\
& z_{2}=\omega \sin (\omega t)+\lambda \operatorname{coth}(\lambda t+\gamma) \cos (\omega t)
\end{aligned}
$$

with $W\left(z_{1}, z_{2}\right)=\omega\left(\omega^{2}+\lambda^{2}\right)$ are fundamental solutions of the following equation:

$$
z^{\prime \prime}+\left(\omega^{2}-\frac{2 \lambda^{2}}{\sinh ^{2}(\lambda t+\gamma)}\right) z=0
$$

and then carry out the substitution $y=z \tanh (\lambda t+\gamma)$. Details are left to the reader. The particular solution of the nonhomogeneous equation can be found by the variation of parameters and/or verified by the substitution. Review of other integrable second-order differential equations is given in [44].

\section{REFERENCES}

[1] K. Andriopoulos and P. G. L. Leach, Wavefunctions for the time-dependent linear oscillator and Lie point symmetries, J. Phys. A 38 (2005) \#20, 4365-4374.

[2] F. Antonsen, Sturmian basis functions for the harmonic oscillator, arXiv:9809062v1 [quant-ph] 9 Feb 2008.

[3] G. P. Arrighini and N. L. Durante, More on the quantum propagator of a particle in a linear potential, Am. J. Phys. 64 (1996) \# 8, 1036-1041.

[4] H. Bateman, On dissipative systems and related variational principles, Phys. Rev. 38 (1931), 815-819.

[5] H. Bateman, Partial Differential Equations of Mathematical Physics, Dover, New York, 1944.

[6] L. A. Beauregard, Propagators in nonrelativistic quantum mechanics, Am. J. Phys. 34 (1966), 324-332.

[7] M. V. Berry, Classical adiabatic angles and quantum adiabatic phase, J. Phys. A: Math. Gen 18 (1985) \# 1, $15-27$.

[8] M. V. Berry and J. Hannay, Classical non-adiabatic angles, J. Phys. A: Math. Gen 21 (1988) \# 6, L325-L331.

[9] C. Bertoni, F. Finelli and G. Venturi, Adiabatic invariants and scalar fields in a de Sitter space-time, Phys. Lett. A 237 (1998), 331-336.

[10] N. N. Bogoliubov and D. V. Shirkov, Introduction to the Theory of Quantized Fields, third edition, John Wiley \& Sons, New York, Chichester, Brisbane, Toronto, 1980.

[11] W. E. Brittin, A note on the quantization of dissipative systems, Phys. Rev. 77 (1950), 396-397.

[12] L. S. Brown and Y. Zhang, Path integral for the motion of a particle in a linear potential, Am. J. Phys. 62 (1994) \# 9, 806-808.

[13] P. Caldirola, Forze non conservative nella meccanica quantistica, Nuovo Cim. 18 (1941), 393-400.

[14] P. Camiz, A. Gerardi, C. Marchioro, E. Presutti and E. Scacciatelli, Exact solution of a time-dependent quantal harmonic oscillator with a singular perturbationg, J. Math. Phys. 12 (1971) \#10, 2040-2043. 
[15] J. F. Cariñena, J. de Lucas and M. F. Rañada, A geometric approach to time evolution operators of Lie quantum systems, arXiv:0811.4386v1 [math-ph] 26 Nov 2008.

[16] J. F. Cariñena, J. de Lucas and M. F. Rañada, Lie systems and integrability conditions for -dependent frequency harmonic oscillator, arXiv:0908.2292v1 [math-ph] 17 Aug 2009.

[17] R. M. Cavalcanti, Lie systems and integrability conditions for t-dependent frequency harmonic oscillator, arXiv:9805005v2 [quant-ph] 6 Nov 1998.

[18] J. M. Cerveró and J. D. Lejarreta, SO (2,1)-invariant systems and the Berry phase, J. Phys. A: Math. Gen 22 (1989) \# 14, L663-L666.

[19] V. K. Chandrasekar, M. Senthilvelan and M. Lakshmann, On the Lagrangian and Hamiltonian description of the damped linear harmonic oscillator, J. Math. Phys. 48 (2007), 032701.

[20] B. K. Cheng, Exact evaluation of the propagator for the damped harmonic oscillator, J. Phys. A 17 (1984) \#12, $2475-2484$.

[21] B. K. Cheng, Extended Feynman formula for damped harmonic oscillator with time-dependent perturbative force, Phys. Lett. A 110 (1985), 347-350.

[22] V. N. Chernega and V. I. Man'ko, The wave function of the classical parametric oscillator and the tomographic probability of the oscillator's state, Journal of Russian Laser Research 29 (2008) \# 4, 347-356.

[23] D. Chruściński, Quantum damped oscillator II: Bateman's Hamiltonian vs. $2 D$ parabolic potential barrier, Ann. Phys. 321 (2006), 840-853.

[24] D. Chruściński and J. Jurkowski, Quantum damped oscillator I: dissipation and resonances, Ann. Phys. 321 (2006), 854-874.

[25] D. Chruściński and J. Jurkowski, Memory in a nonlocally damped oscillator, arXiv:0707.1199v2 [quant-ph] 7 Dec 2007.

[26] R. Cordero-Soto, R. M. Lopez, E. Suazo and S. K. Suslov, Propagator of a charged particle with a spin in uniform magnetic and perpendicular electric fields, Lett. Math. Phys. 84 (2008) \#2-3, 159-178.

[27] R. Cordero-Soto, E. Suazo and S. K. Suslov, Models of damped oscillators in quantum mechanics, Journal of Physical Mathematics, 1 (2009), S090603 (16 pages).

[28] R. Cordero-Soto and S. K. Suslov, Time reversal for modified oscillators, Theoretical and Mathematical Physics 162 (2010) \#3, 286-316; see also arXiv:0808.3149v9 [math-ph] 8 Mar 2009.

[29] E. D. Courant and H. S. Snyder, Theory of the alternating-gradient synchrotron, Ann. Phys. (N. Y.) 3 (1958), $1-48$.

[30] F. Dalfovo, S. Giorgini, L. P. Pitaevskii and S. Stringari, Theory of Bose-Einstein condensation in trapped gases, Rev. Mod. Phys. 71 (1999), 463-512.

[31] A. Degasperis and S. N. M. Ruijsenaars, Newton-equivalent Hamiltonians for the harmonic oscillator, Ann. Phys. 293 (2001), 92-109.

[32] H. H. Denman, On linear friction in Lagrange's equation, Am. J. Phys. 34 (1966) \# 12, 1147-1149.

[33] H. Dekker, Classical and quantum mechanics of the damped harmonic oscillator, Phys. Rep. 80 (1981), 1-112.

[34] F. Delgado C., B. Mielnik and M. A. Reyes, Squeezed states and Helmholtz spectra, Phys. Lett. A 237 (1998) \#6, 359-364.

[35] G. Ditto and F. J. Turrubiates, The damped harmonic oscillator in deformation quantization, Phys. Lett. A 352 (2006), 309-316.

[36] V. V. Dodonov, I. A. Malkin and V. I. Man'ko, Integrals of motion, Green functions, and coherent states of dynamical systems, Int. J. Theor. Phys. 14 (1975) \# 1, 37-54.

[37] V. V. Dodonov and V. I. Man'ko, Coherent states and resonance of a quantum damped oscillator, Phys. Rev. A 20 (1979) \# 2, 550-560.

[38] V. V. Dodonov and V. I. Man'ko, Generalizations of uncertainty relations in quantum mechanics, in: Invariants and the Evolution of Nonstationary Quantum Systems, Proceedings of Lebedev Physics Institute, vol. 183, pp. 5-70, Nauka, Moscow, 1987 [in Russian].

[39] V. V. Dodonov and V. I. Man'ko, Invariants and correlated states of nonstationary quantum systems, in: Invariants and the Evolution of Nonstationary Quantum Systems, Proceedings of Lebedev Physics Institute, vol. 183, pp. 71-181, Nauka, Moscow, 1987 [in Russian]; English translation published by Nova Science, Commack, New York, 1989, pp. 103-261.

[40] A. V. Dodonov, S. S. Mizrahi and V. V. Dodonov, Quantum master equations from classical Lagrangians with two stochastic forces, Phys. Rev. E 75 (2007), 011132 (10 pages). 
[41] E. V. Doktorov, I. A. Malkin and V. I. Man'ko, Dynamical symmetry of vibronic transitions in polyatomic molecules and Frank-Condon principle, J. Mol. Spectrosc. 64 (1977), 302-326.

[42] P. Ehrenfest, Bemerkung über die angenäherte Gültigkeit der klassischen Mechanik innerhalb der Quantenmechanik, Zeitschrift für Physik A 45 (1927), 455-457.

[43] C. J. Eliezer and A. Grey, A note on the time-dependent harmonic oscillator, SIAM J. Appl. Math. 30 (1976) \#3, 463-468.

[44] V. P. Ermakov, Second-order differential equations. Conditions of complete integrability, Universita Izvestia Kiev, Series III 9 (1880), 1-25; see also Appl. Anal. Discrete Math. 2 (2008) \#2, 123-145 for English translation of Ermakov's original paper.

[45] L. D. Faddeev, Feynman integrals for singular Lagrangians, Theoretical and Mathematical Physics 1 (1969) \#1, 3-18 [in Russian].

[46] R. P. Feynman, The Principle of Least Action in Quantum Mechanics, Ph. D. thesis, Princeton University, 1942; reprinted in: "Feynman's Thesis - A New Approach to Quantum Theory", (L. M. Brown, Editor), World Scientific Publishers, Singapore, 2005, pp. 1-69.

[47] R. P. Feynman, Space-time approach to non-relativistic quantum mechanics, Rev. Mod. Phys. 20 (1948) \# 2, 367-387; reprinted in: "Feynman's Thesis - A New Approach to Quantum Theory", (L. M. Brown, Editor), World Scientific Publishers, Singapore, 2005, pp. 71-112.

[48] R. P. Feynman, The theory of positrons, Phys. Rev. 76 (1949) \# 6, 749-759.

[49] R. P. Feynman, Space-time approach to quantum electrodynamics, Phys. Rev. 76 (1949) \# 6, 769-789.

[50] R. P. Feynman and A. R. Hibbs, Quantum Mechanics and Path Integrals, McGraw-Hill, New York, 1965.

[51] F. Finelli, A. Gruppuso and G. Venturi, Quantum fields in an expanding universe, Class. Quantum Grav. 16 (1999), 3923-3935.

[52] F. Finelli, G. P. Vacca and G. Venturi, Chaotic inflation from a scalar field in nonclassical states, Phys. Rev. D 58 (1998), 103514 (14 pages).

[53] K. Gottfried and T.-M. Yan, Quantum Mechanics: Fundamentals, second edition, Springer-Verlag, Berlin, New York, 2003.

[54] I. C. Goyal, R. L. Gallawa and A. K. Ghatak, Accuracy of eigenvalues: a comparison of two models, J. Math. Phys. 34 (1993) \#3, 1169-1175.

[55] G. A. Hagedorn, M. Loss and J. Slawny, Non-stochasticity of time-dependent quadratic Hamiltonians and spectra of canonical transformations, J. Phys. A: Math. Gen. 19 (1986), 521-531.

[56] G. A. Hagedorn, Raising and lowering operators for semiclassical wave package, Ann. Phys. 269 (1998), 77-104.

[57] J. H. Hannay, Angle variable holonomy in adiabatic excursion of an integrable Hamiltonian, J. Phys. A: Math. Gen 18 (1985) \# 2, 221-230.

[58] R. M. Hawkins and J. E. Lidsey, Ermakov-Pinney equation in scalar field cosmologies, Phys. Rev. D 66 (2002), 023523 (8 pages).

[59] E. Hille, Lectures on Ordinary Differential Equations, Addison-Wesley, Reading, 1969.

[60] B. R. Holstein, The linear potential propagator, Am. J. Phys. 65 (1997) \#5, 414-418.

[61] B. R. Holstein, The harmonic oscillator propagator, Am. J. Phys. 67 (1998) \#7, 583-589.

[62] K. Husimi, Miscellanea in elementary quantum mechanics, I, Prog. Theor. Phys. 9 (1953) \#3, 238-244.

[63] K. Husimi, Miscellanea in elementary quantum mechanics, II, Prog. Theor. Phys. 9 (1953) \#4, 381-402.

[64] F. Iacob, Relativistic pseudo-Gaussian oscillators, Phys. Lett. A 374 (2010) \#11-12, 1332-1335.

[65] E. V. Ivanova, I. A. Malkin and V. I. Man'ko, Coherent states and radiation of non-stationary quadatic systems, Phys. Lett. A. 50 (1974) \#1, 23-24.

[66] Yu. Kagan, E. L. Surkov and G. V. Shlyapnikov, Evolution of Bose-condensed gas under variations of the confining potential, Phys. Rev. A 54 (1996) \#3, R1753-R1756.

[67] Yu. Kagan, E. L. Surkov, and G. V. Shlyapnikov, Evolution of Bose gas in anisotropic time-dependent traps, Phys. Rev. A 55 (1997) \#1, R18-R21.

[68] A. Kamenshchik, M. Luzzi and G. Venturi, Remarks on the methods of comparison equations (generalized WKB method) and the generalized Ermakov-Pinney equation, arXiv:0506017v2 [math-ph] 9 Feb 2006.

[69] E. Kanai, On the quantization of dissipative systems, Prog. Theor. Phys. 3 (1948), 440-442.

[70] A. G. Karavayev, Trajectory-coherent states for the Caldirola-Kanai Hamiltonian, arXiv:9709009v1 [quant-ph] 4 Sep 1997. 
[71] F. Kheirandish and M. Amooshahi, Dissipation in quantum mechanics, scalar and vector field theory, arXiv:0610133v1 [quant-ph] 17 Oct 2006.

[72] S. P. Kim, A. F. Santana and F. C. Khanna, Decoherence of quantum damped oscillators, arXiv:0202089v1 [quant-ph] 15 Feb 2002.

[73] Yu. S. Kivshar, T. J. Alexander, and S. K. Turitsyn, Nonlinear modes of a macroscopic quantum oscillator, Phys. Lett. A 278 (2001) \#1, 225-230.

[74] J. R. Klauder and E. C. G. Sudarshan, Fundamental of Quantum Optics, Benjamin, New York, 1968.

[75] D. Kochan, Quantization of equations of motion, Acta Polytechnica 47 (2007) \#2-3, 60-67.

[76] D. Kochan, Direct quantization of equations of motion: from classical dynamics to transition amplitudes via strings, arXiv:0703073 [hep-th] 8 Mar 2007.

[77] D. Kochan, Functional integral for non-Lagrangian systems, Phys. Rev. A 81 (2010) \#2, 022112.

[78] M. Kruskal, Asymptotic theory of Hamiltonian and other systems with all solutions nearly periodic, J. Math. Phys. 3 (1962), 806-828.

[79] L. D. Landau and E. M. Lifshitz, Mechanics, Pergamon Press, Oxford, 1976.

[80] L. D. Landau and E. M. Lifshitz, Quantum Mechanics: Nonrelativistic Theory, Pergamon Press, Oxford, 1977.

[81] N. Lanfear and S. K. Suslov, The time-dependent Schrödinger equation, Riccati equation and Airy functions, arXiv:0903.3608v 5 [math-ph] 22 Apr 2009.

[82] P. G. L. Leach, Note on the time-dependent damped and forced harmonic oscillator, Am. J. Phys. 46 (1978) \#12, 1247-1249.

[83] P. G. L. Leach, On a generalization of the Lewis invariant for the time-dependent harmonic oscillator, SIAM J. Appl. Math. 34 (1978) \#3, 496-503.

[84] P. G. L. Leach, Berry's phase and wave functions for time-dependent Hamiltonian systems, J. Phys. A: Math. Gen 23 (1990), 2695-2699.

[85] P. G. L. Leach and K. Andriopoulos, The Ermakov equation: a commentary, Appl. Anal. Discrete Math. 2 (2008) \#2, 146-157.

[86] P. G. L. Leach and K. Andriopoulos, An invariant for the doubly generalized classical Ermakov-Pinney system and its quantal equivalent, Phys. Scripta 77 (2008), 015002 (7 pages).

[87] P. G. L. Leach, A. Karasu (Kalkanli), M. C. Nucci and K. Andriopoulos, Ermakov's superintegrable toy and nonlocal symmetries, Symmetry, Integrability and Geometry: Methods and Applications, SIGMA 1 (2005), 018 (15 pages).

[88] H. R. Lewis, Jr., Classical and quantum systems with time-dependent harmonic-oscillator-type Hamiltonians, Phys. Rev. Lett. 18 (1967) \#13, 510-512.

[89] H. R. Lewis, Jr., Motion of a time-dependent harmonic oscillator, and of a charged particle in a class of time-dependent, axially symmetric electromagnetic fields, Phys. Rev. 172 (1968) \#5, 1313-1315.

[90] H. R. Lewis, Jr., Class of exact invariants for classical and quantum time-dependent harmonic oscillators, J. Math. Phys. 9 (1968) \#11, 1976-1986.

[91] H. R. Lewis, Jr., and W. B. Riesenfeld, An exact quantum theory of the time-dependent harmonic oscillator and of a charged particle in a time-dependent electromagnetic field, J. Math. Phys. 10 (1969) \#8, 1458-1473.

[92] E. H. Lieb and M. Loss, Analysis, 2nd. ed, Grad. Stud. Math. 14, AMS, Providence, RI, 2001.

[93] R. M. Lopez and S. K. Suslov, The Cauchy problem for a forced harmonic oscillator, Revista Mexicana de Física, 55 (2009) \#2, 195-215; see also arXiv:0707.1902v8 [math-ph] 27 Dec 2007.

[94] F. G. Major, V. N. Gheorghe and G. Werth, Charged Particle Traps, Springer-Verlag, Berlin, Heidelberg, 2005.

[95] I. A. Malkin and V. I. Man'ko, Dynamical Symmetries and Coherent States of Quantum System, Nauka, Moscow, 1979 [in Russian].

[96] I. A. Malkin, V. I. Man'ko and D. A. Trifonov, Invariants and the evolution of coherent states for a charged particle in a time-dependent magnetic field, Phys. Lett. A. 30 (1969) \#7, 414.

[97] I. A. Malkin, V. I. Man'ko and D. A. Trifonov, Coherent states and transition probabilities in a time-dependent electromagnetic field, Phys. Rev. D. 2 (1970) \#2, 1371-1385.

[98] I. A. Malkin, V. I. Man'ko and D. A. Trifonov, Linear adiabatic invariants and coherent states, J. Math. Phys. 14 (1973) \#5, 576-582.

[99] A. Mandilara, E. Karpov and N. J. Cerf, Non-Gaussianity bounded uncertainty relation for mixed states, arXiv:0910.3474v1 [quant-ph] 19 Oct 2009. 
[100] V. P. Maslov and M. V. Fedoriuk, Semiclassical Approximation in Quantum Mechanics, Reidel, Dordrecht, Boston, 1981.

[101] M. Meiler, R. Cordero-Soto and S. K. Suslov, Solution of the Cauchy problem for a time-dependent Schrödinger equation, J. Math. Phys. 49 (2008) \#7, 072102: 1-27; published on line 9 July 2008, URL: http://link.aip.org/link/?JMP/49/072102, see also arXiv: 0711.0559v4 [math-ph] 5 Dec 2007.

[102] E. Merzbacher, Quantum Mechanics, third edition, John Wiley \& Sons, New York, 1998.

[103] W. E. Milne, The numerical determination of characteristic numbers, Phys. Rev. 35 (1930) \#7, $863-867$.

[104] M. Montesinos, Heisenberg's quantization of dissipative systems, Phys. Rev. A 68 (2003), 014101.

[105] D. A. Morales, Correspondence betweem Berry's phase and Lewis's phase for quadratic Hamiltonians, J. Phys. A: Math. Gen 21 (1988) \# 18, L889-L892.

[106] A. Mostafazadeh, Time-dependent diffeomorphisms as quantum canonical transformations and time-dependent harmonic oscillator, arXiv:9807002v1 [quant-ph] 1 Jul 1998.

[107] C. A. Muñoz, J. Rueda-Paz and K. B. Wolf, Discrete repulsive oscillator wavefunctions, J. Phys. A: Math. Theor. 42 (2009), 485210 (12pp).

[108] P. Nardone, Heisenberg picture in quantum mechanics and linear evolutionary systems, Am. J. Phys. 61 (1993) \# 3, 232-237.

[109] J. von Neumann, Mathematical Foundation of Quantum Mechanics, Princeton University Press, Princeton, New Jersey, 1983.

[110] M. M. Nieto and D. R. Truax, Time-dependent Schrödinger equations having isomorphic symmetry algebras. I. Classes of interrelated equations, e-Preprint LA-UR-98-727 (quant-ph/9811075) 1 Feb 2008.

[111] A. F. Nikiforov, S. K. Suslov and V. B. Uvarov, Classical Orthogonal Polynomials of a Discrete Variable, Springer-Verlag, Berlin, New York, 1991.

[112] H. G. Oh, H. R. Lee and T. F. George, Exact wave functions and coherent states of a damped driven harmonic oscillator, Phys. Rev. A 39 (1989) \# 11, 5515-5522.

[113] Y-G. Oh, Cauchy problem and Ehrenfest's law of nonlinear Schrödinger equations with potentials, J. Differential Equations 81 (1989), 255-274.

[114] P. B. E. Padilla, Ermakov-Lewis dynamic invariants with some applications, arXiv:0002005v3 [math-ph] 18 Mar 2000.

[115] I. A. Pedrosa and I. Guedes, Quantum states of a generalized time-dependent inverted harmonic oscillator, arXiv:030708v1 [quant-ph] 10 Jul 2003.

[116] J. P. Reithmaier et al, Size dependence of confined optical modes in photonic quantum dots, Phys. Rev. Lett. 78 (1997) \#2, 378-381.

[117] A. M. Perelomov and V. S. Popov, Group-theoretical aspects of the variable frequency oscillator problem, Theoretical and Mathematical Physics 1 (1969) \#34, 275-285.

[118] A. M. Perelomov and Ya. B. Zeldovich, Quantum Mechanics: Selected Topics, World Scientific Publishing Co., Inc., River Edje, NJ, 1998.

[119] V. M. Pérez-García, P. Torres and G. D. Montesinos, The method of moments for nonlinear Schrödinger equations: theory and applications, SIAM J. Appl. Math. 67 (2007) \#4, 990-1015.

[120] E. Pinney, The nonlinear differential equation $y^{\prime \prime}+p(x) y+c y^{-3}=0$, Proc. Am. Math. Soc. 1 (1950), 681.

[121] V. S. Popov and A. M. Perelomov, Parametric excitation of a quantum oscillator, Soviet Physics JETP 29 (1969) \# 4, 738-745.

[122] V. S. Popov and A. M. Perelomov, Parametric excitation of a quantum oscillator, II, Soviet Physics JETP 30 (1969) \# 5, 910-913.

[123] R. W. Robinett, Quantum mechanical time-development operator for the uniformly accelerated particle, Am. J. Phys. 64 (1996) \#6, 803-808.

[124] H. Rosu and P. Espinoza, An Ermakov study of $Q \neq 0$ EFRW minisuperspace oscillators, III Workshop of DGFM/SMF, Nov. 28-Dec. 3, 1999, León, Gto., Mexico; arXiv:9912033v1 [gr-qc] 9 Dec 1999.

[125] H. Rosu, P. Espinoza and M. Reyes, Ermakov approach for $Q=0$ empty FRW minisuperspace oscillators, Nuovo Cimento B 114 (1999), 1435-1440.

[126] M. Ryan, Hamiltonian Cosmology, Springer-Verlag, Berlin, 1972.

[127] S. S. Safonov, Caldirola-Kanai oscillator in classical formulation of quantum mechanics, arXiv:9802057v1 [quant-ph] 23 Feb 1998.

[128] M. Sargent III, M. O. Scully and W. E. Lamb, Jr., Laser Physics, Addison-Wesley, Reading, 1974. 
[129] L. I. Schiff, Quantum Mechanics, third edition, McGraw-Hill, New York, 1968.

[130] D. Schuch, Riccati and Ermakov equations in time-dependent and time-independent quantum systems, Symmetry, Integrability and Geometry: Methods and Applications, SIGMA 4 (2008), 043 (16 pages).

[131] M. O. Scully and M. S. Zubairy, Quantum Optics, Cambridge University Press, Cambridge, 1997.

[132] Yu. F. Smirnov and K. V. Shitikova, The method of K harmonics and the shell model, Soviet Journal of Particles \& Nuclei 8 (1977) \#4, 344-370.

[133] S. Solimeno, B. Grosignani and P. DiPorto, Quantum harmonic oscillator with time-dependent frequency, J. Math. Phys. 10 (1969) \#10, 1922-1928.

[134] E. Suazo, On Schrödinger equation with time-dependent quadratic Hamiltonians in $\mathbb{R}^{d}$, arXiv:0912.2113v2 [math-ph] 11 Dec 2009.

[135] E. Suazo and S. K. Suslov, An integral form of the nonlinear Schrödinger equation with wariable coefficients, arXiv:0805.0633v2 [math-ph] 19 May 2008.

[136] E. Suazo and S. K. Suslov, Cauchy problem for Schrödinger equation with variable quadratic Hamiltonians, under preparation.

[137] E. Suazo, S. K. Suslov and J. M. Vega, The Riccati differential equation and a diffusion-type equation, arXiv:0807.4349v4 [math-ph] 8 Aug 2008.

[138] S. K. Suslov, Dynamical invariants for variable quadratic Hamiltonians, Physica Scripta, to appear; see also arXiv:1002.0144v5 [math-ph] 26 Feb 2010.

[139] I. R. Svin'in, Quantum-mechanical description of friction, Theoretical and Mathematical Physics 22 (1975) \# $1,67-75$.

[140] I. R. Svin'in, Quantum description of Brownian motion in an external field, Theoretical and Mathematical Physics 27 (1976) \# 2, 478-483.

[141] K. R. Symon, The adiabatic invariant of the linear and nonlinear oscillator, J. Math. Phys. 11 (1970) \#4, $1320-1330$.

[142] L. V. Tarasov, Laser Physics, Mir Publishers, Moscow, 1983.

[143] V. E. Tarasov, Quantization of non-Hamiltonian and dissipative systems, Phys. Lett. A 288 (2001), 173-182.

[144] N. S. Thomber and E. F. Taylor, Propagator for the simple harmonic oscillator, Am. J. Phys. 66 (1998) \# 11, $1022-1024$.

[145] Y. Tikochinsky, Exact propagators for quadratic Hamiltonians, J. Math. Phys. 19 (1978) \#4, 888-891.

[146] Ch-I. Um, K-H. Yeon and T. F. George, The quantum damped oscillator, Phys. Rep. 362 (2002), 63-192.

[147] G. Velo, Mathematical aspects of the nonlinear Schrödinger equation, in: Nonlinear Klein-Gordon and Schrödinger Systems: Theory and Applications (L. Vázquez, L. Streit and V. P. Pérez-Garsia, Editors), World Scientific, Singapore, 1996, pp. 39-67.

[148] D. F. Walls and G. J. Millburn, Quantum Optics, Springer-Verlag, Heidelberg, 1994.

[149] K. B. Wolf, On time-dependent quadratic Hamiltonians, SIAM J. Appl. Math. 40 (1981) \#3, 419-431.

[150] K-H. Yeon, K-K. Lee, Ch-I. Um, T. F. George and L. N. Pandey, Exact quantum theory of a time-dependent bound Hamiltonian systems, Phys. Rev. A 48 (1993) \# 4, 2716-2720.

[151] K-H. Yeon, Ch-I. Um and T. F. George, Coherent states for the damped oscillator, Phys. Rev. A 36 (1987) \# 11, $5287-5291$.

Mathematical, Computational and Modeling Sciences Center, Arizona State University, Tempe, AZ 85287-1804, U.S.A.

E-mail address: ricardojavier81@gmail.com

Department of Mathematical Sciences, University of Puerto Rico, Mayaquez, call box 9000, PR 00681-9000, Puerto Rico

E-mail address: erwin.suazo@upr.edu

School of Mathematical and Statistical Sciences \& Mathematical, Computational and Modeling Sciences Center, Arizona State University, Tempe, AZ 85287-1804, U.S.A.

E-mail address: sks@asu.edu

URL: http://hahn.la.asu.edu/ ${ }^{\sim}$ suslov/index.html 